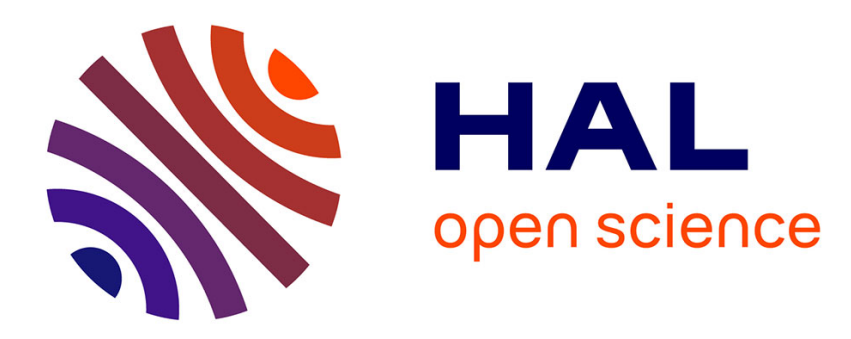

\title{
Phase resetting for a network of oscillators via phase response curve approach
}

\author{
Denis Efimov
}

\section{To cite this version:}

Denis Efimov. Phase resetting for a network of oscillators via phase response curve approach. Biological Cybernetics (Modeling), 2015, 109 (1), pp.95-108. 10.1007/s00422-014-0629-z . hal-01061988

\section{HAL Id: hal-01061988 \\ https://hal.inria.fr/hal-01061988}

Submitted on 8 Sep 2014

HAL is a multi-disciplinary open access archive for the deposit and dissemination of scientific research documents, whether they are published or not. The documents may come from teaching and research institutions in France or abroad, or from public or private research centers.
L'archive ouverte pluridisciplinaire HAL, est destinée au dépôt et à la diffusion de documents scientifiques de niveau recherche, publiés ou non, émanant des établissements d'enseignement et de recherche français ou étrangers, des laboratoires publics ou privés. 


\title{
PHASE RESETTING FOR A NETWORK OF OSCILLATORS VIA PHASE RESPONSE CURVE APPROACH
}

\author{
Efimov D. ${ }^{1,2,3}$
}

The problem of phase regulation for a population of oscillating systems is considered. The proposed control strategy is based on a Phase Response Curve (PRC) model of an oscillator (the first order reduced model obtained for linearized system and inputs with infinitesimal amplitude). It is proven that the control provides phase resetting for the original nonlinear system. Next the problem of phase resetting for a network of oscillators is considered when applying a common control input. Performance of the obtained solutions is demonstrated via computer simulation for three different models of circadian/neural oscillators.

Keywords: oscillation control; network of oscillators; phase response curve.

\section{Introduction}

Many systems in biology, physics, chemistry and engineering perform periodic oscillations in the main operating mode [B, I, K, MMP, PRK, W]. This is why the interest in analysis and synthesis of such systems is continuously growing during the last decades [FP, GH, S]. Any periodic oscillating mode can be characterized by its frequency (or frequency spectrum), phase and amplitude. Each of these characteristics can be controlled [ABS, Be, EF, Ko, Ku]. The problem of phase or frequency resetting consists of assignment of desired values for phase and frequency applying some (maybe periodic) control input [B, GGS, W]. Frequently in biological applications there are restrictions on the form and amplitude of the control available for resetting, which prevent application of the conventional control approaches. This problem is also addressed in the oscillator synchronization framework, when external input is just an output of another oscillator, whose phase and frequency become desired for the resetting [PRK]. If it is necessary to reset both frequency and phase, then this problem is called entrainment [I, PRK].

In this work we will focus our attention on phase resetting problem only [T]. There exist several approaches to the problem solution. The first one is based on master-slave synchronization theory, when the master oscillator attempts to entrain its own phase of the slave one. This approach is very promising, but it assumes rather serious modifications of slave equations. Frequently a similarity of both systems and measurements of all states are required. The approach is suitable for synthesis of technical systems [Be, $\mathrm{Ku}$ ], but it meets serious obstructions for application in biology, physics or chemistry. Another line of research deals with an optimal or predictive control application for phase resetting [BSD1, BSD2, FoP]. These methods require availability of the full exact information about the oscillator model and its coefficients, and these requirements cause difficulty for application. The third approach uses an assumption on weak coupling/excitation, i.e. it restricts the amplitude of external inputs [PRK], which is a mild or natural constraint in some applications. The last approach is based on PRC application and Poincaré phase map approach [Gl, GG, I, KS]. The advantage of this control approach [E11, ESS, DM, LDR] consists in low dimension of PRC (it is a scalar map of scalar argument, which completely describes phase resetting caused by a "pulse" input). Additionally, the PRC can be measured experimentally even for oscillators for which detailed models are not known.

In some cases the phase dynamics appear for a network or a population of oscillators. The colony of the smallest free-living eukaryotes [TPM] or the mammalian circadian pacemaker neural network [Z, AFFP], networks of neural

\footnotetext{
${ }^{1}$ Non-A project @ Inria, Parc Scientifique de la Haute Borne, 40 avenue Halley, 59650 Villeneuve d'Ascq, France.

${ }^{2}$ LAGIS UMR 8219, Ecole Centrale de Lille, Avenue Paul Langevin, 59651 Villeneuve d'Ascq, France.

${ }^{3}$ Department of Control Systems and Informatics, University ITMO, 49 avenue Kronverkskiy, 197101 Saint Petersburg, Russia.

This work was partially supported by the Government of Russian Federation (Grant 074-U01) and the Ministry of Education and Science of Russian Federation (Project 14.Z50.31.0031).
} 
oscillators [CA, SEW, T1] or wireless networks [WND] can be considered as examples of such a network. Despite the existence of many works devoted to the problem of the phase synchronization in networks of oscillators [I, K, MMP, PRK, W], the controlled phase resetting is not well studied. In this work we will consider the case when an average phase of the population can be measured or estimated and there is a common control input applied to all oscillators in the group. It is assumed that there is no coupling between oscillators (or the coupling effect is very weak comparing with the control influence; if the coupling is important, then the entire coupled system can be considered as an oscillator). For example, in the case of [TPM] due to a small size it is impossible to apply control or measure the state of a single organism, in [Z, AFFP] the pacemaker has to be analyzed and treated as whole. In this work we will assume that all oscillators have similar models (the same PRC). The initial phases of oscillators are distributed around the measured average one with some bounded deviation. The objective is to reset all oscillators via the common input with simultaneous synchronization of all uncoupled oscillators, i.e. after the resetting all systems in the network should oscillate with the same desired phase.

It is worth noting that the problem of synchronization of a network of isolated (weakly connected) oscillators is rather important from practical and theoretical points of view. The phase synchronization phenomenon for isolated oscillators is considered in [W] (Chapter 4). Synchronization of two uncoupled lasers by a common excitation is investigated in [RT]. The occurrence of synchronous motoneuron discharge in the muscles of human performing steady isometric contractions is studied in [DS, SIF] (this well-known observable fact may be interpreted as synchronization of uncoupled oscillators). In population ecology, Moran's theorem establishes that the time correlation of two isolated populations of the same species is equal to the correlation between the environmental variability (i.e. the error in the common input of both populations is proportional to the synchronization error) [Mo]. This Moran's effect is experimentally verified in a number of cases [RHK, KoK, PW, CDST]. Another example is a population of bivalves, whose rhythmic activity is driven by environmental forces [Tr]. The problem of phase synchronization between uncoupled oscillators driven by a common stochastic external forcing is widely studied, see [NAN] and references therein. The problem of an optimal control design for an ensemble of decoupled neural oscillators is considered in [LDR].

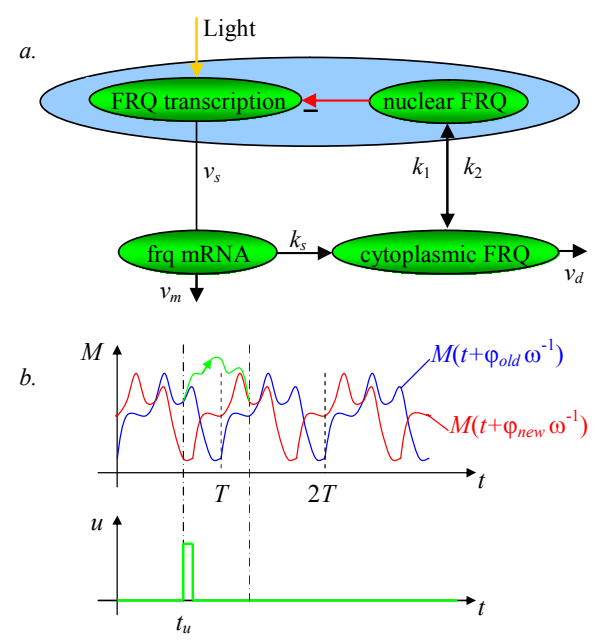

Fig. 1. Circadian oscillations in Neurospora: (a) model and (b) phase resetting by a pulse (blue line represents behavior of $M(t)$ before the pulse $u(t)$, red line corresponds to oscillation of $M(t)$ after the pulse, green line is the transient motion caused by the pulse). 
To achieve the posed synchronization goals, in this work we are going to extend the results of [DM, ESS, E11] for phase resetting by infinitesimal pulse inputs. A modification of the control strategy proposed in [ESS, E11] for a single oscillator is given. The control determines the pulse timing based on the PRC. The PRC map is calculated for the system linearized around the limit cycle and inputs with small amplitudes. It is proven that this control ensures phase resetting for the original nonlinear oscillating system, which is a contribution of this work. Next, this control strategy is developed for application in populations of oscillators. The proposed controls are applied for circadian oscillators of third and tenth orders, and to a network of neurons forming a mammalian circadian pacemaker.

More detailed problem statement and motivation are given in section 2. Some preliminaries about phase dynamics are summarized in section 3. The control synthesis for a single oscillator is presented in section 4 . Section 5 contains the proof that the control designed for a simplified PRC model of an oscillator makes the phase resetting for the original nonlinear system. The control extension on the phase resetting for a population of oscillators is given in Section 6 . Applications are considered in section 7.

\section{Motivation and problem statement}

Many living organisms, from bacteria to mammals, display circadian rhythms. These are sustained oscillations with a period close to 24 hours. Consider a simple biological model of circadian oscillations in Neurospora [LGG] presented in Fig. 1,a. Corresponding equations of mathematical model have form

$$
\begin{aligned}
& \dot{M}=\left(v_{s}+u\right) \frac{K_{I}^{n}}{K_{I}^{n}+F_{N}^{n}}-v_{m} \frac{M}{K_{m}+M} ; \\
& \dot{F}_{C}=k_{s} M-v_{d} \frac{F_{C}}{K_{d}+F_{C}}-k_{1} F_{C}+k_{2} F_{N} ; \\
& \dot{F}_{N}=k_{1} F_{C}-k_{2} F_{N},
\end{aligned}
$$

where the variables $M>0, F_{C}>0, F_{N}>0$ denote the concentrations (defined with respect to the total cell volume) of the frq mRNA, the cytoplasmic and nuclear forms of FRQ respectively. The parameter $v_{s}$ defines the rate of frq transcription (this parameter increases in the light phase), the influence of light (the external input in the model (1)) is denoted by $u$. Description of other parameters appearing in these equations can be found in [LGG], the following values of parameters are proposed in [LGG]:

$$
v_{m}=0.505, v_{d}=1.4, k_{s}=0.5, k_{1}=0.5, k_{2}=0.6 K_{M}=0.5, K_{I}=1, K_{d}=0.13, n=4,1 \leq v_{s}+u \leq 2.5 .
$$

For all these values the system (1) has single unstable equilibrium and globally attractive limit cycle. For different values of the parameter $1 \leq v_{s} \leq 2.5$ the system (1) has different periods $18.87 \leq T \leq 25.2$ on the limit cycle (as well as different amplitudes of oscillations).

Phase resetting in (1) is carried out by the light stimulus $u$. It is well known that periodic excitation by light input leads to phase and frequency entrainment of the natural circadian oscillations [LGG, T]. The question arises: can we design such a light excitation signal providing phase resetting to a desired phase? Such input $u$ is usually chosen as a periodic square pulse [BSD1, BSD2, LGG] with a period close to $T$ (where $T$ is the period of (1)), when it does not influence the system frequency.

This problem has practical importance and many travelers are familiar with "jet-lag" problem caused by circadian rhythm disorder after long distance flights. Organisms need some time to adapt their phase of internal circadian rhythms to new environmental light conditions. Improvement of the transient time as well as an advance resetting of the desired phase is of great importance in this way. Light therapy is recognized as one of the main methods for phase resetting.

In the works [DM, ESS, E11] the approaches for design of a phase resetting control $u$ based on PRC have been proposed. In biology, the PRC is recognized as one of the main tools for phase resetting investigation [I]. By definition, 
the PRC tabulates differences in phase caused by a single pulse input. Namely, let $\varphi(t)=\omega t+\varphi_{\text {old }}$ be the current phase of oscillations on the limit cycle (see the illustration in Fig. 1,b, blue curve $M\left(t+\varphi_{\text {old }} \omega^{-1}\right)$ represents the corresponding values of frq mRNA concentration), where $\omega=2 \pi / T$ is the frequency of oscillations and $\varphi_{\text {old }} \in[0,2 \pi)$ is an initial phase. The pulse input moves the system trajectory from the cycle at time instant $t_{u}$. If the input amplitude is not destructive, then this trajectory returns back to the limit cycle and continues to oscillate in general with a new phase $\varphi(t)=\omega t+\varphi_{\text {new }}, \varphi_{\text {new }} \in[0,2 \pi)$ (i.e. the red curve $\left.M\left(t+\varphi_{\text {new }} \omega^{-1}\right)\right)$. This new value $\varphi_{\text {new }}$ depends on the phase of

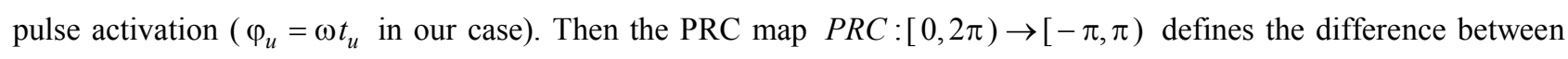
$\varphi_{\text {new }}$ and $\varphi_{\text {old }}$ :

$$
\varphi_{\text {new }}=\varphi_{\text {old }}+\operatorname{PRC}\left(\varphi_{u}\right)
$$

where the PRC argument $0 \leq \varphi_{u}<2 \pi$ fixes the phase of the pulse feeding. If a $T$-periodic pulse train affects the system, then the phase dynamics are described by Poincaré phase map [I]:

$$
\varphi_{i+1}=\varphi_{i}+\operatorname{PRC}\left(\varphi_{i}\right)
$$

where $i \geq 0$ is the number of the current pulse and $\varphi_{i}=\varphi(i T)-\omega i T$. The advantage of the model (2) with respect to (1) consists in its simplicity, it is the first order discrete model and the phase dynamics of any nonlinear oscillator can be reduced to (2) in a similar way.

The phase model (2) is an autonomous discrete system which may have attracting equilibria. These equilibria may be far from desired and depend on PRC behavior. The form of PRC is defined by the system and the input characteristics and, suppose that these cannot be adjusted. There is another possibility to control the system (2) by applying proper timing of pulses. If we can assign the phase of pulse activation $\varpi_{i} \in[0,2 \pi), i \geq 0$, then the model (2) can be rewritten as follows:

$$
\varphi_{i+1}=\varphi_{i}+\operatorname{PRC}\left(\varpi_{i}\right) .
$$

The model (3) is a discrete integrator actuated by PRC control $\varpi$. Modern control theory has many approaches for stabilization of system (3) in a desired position. Several approaches for such control $\varpi$ design have been proposed in [DM, ESS, E11, LDR]. In this work we are going to prove that the controls designed in [ESS] for the simplified model (3) may ensure the phase resetting problem solution for the original nonlinear system (1).

In the case of a population of oscillators, we obtain the model (3) for each oscillator:

$$
\varphi_{i+1}^{j}=\varphi_{i}^{j}+\operatorname{PRC}\left(\varpi_{i}\right), j=1, \ldots, S,
$$

where $S>0$ is the number of oscillators in the population, $\varphi_{i}^{j}$ is the phase of the $j$-th oscillator after the $i$-th pulse activation. The entire population has the same input $\varpi_{i}$ and the measured average phase is

$$
y_{i}=S^{-1} \sum_{j=1}^{S} \varphi_{i}^{j} .
$$

It is necessary to design the input $\varpi_{i}$ resetting the phases of all oscillators to a desired value. This is a typical problem that can appear in the experiments with the colony of Picoeucaryote Ostreococcus tauri in [TPM] or with a mammalian circadian pacemaker $[\mathrm{Z}, \mathrm{AFFP}]$.

\section{Deriving a phase model in vicinity of a stable limit cycle}

Details of the standard procedure for a phase model derivation for an oscillator can be found in [B, I, PRK]. 


\section{A. Linearised model}

Consider a (smooth) dynamical system

$$
\dot{\mathbf{x}}=\mathbf{f}(\mathbf{x}, u), \mathbf{x} \in R^{n}, u \in[-U, U], U>0
$$

and assume that for $u(t) \equiv 0, t \geq 0$ the system (4) has (non-constant) $T$-periodic solution $\gamma(t)=\gamma(t+T) \in R^{n}, t \geq 0$. Then the corresponding limit cycle, described by the set $\Gamma=\left\{\mathbf{x} \in R^{n}: \mathbf{x}=\gamma(t), 0 \leq t<T\right\}$, attracts a non-empty open bounded set of initial conditions $\mathcal{A} \subset R^{n}, \Gamma \subset \mathcal{A}$, and the linearised system

$$
\begin{gathered}
\delta \dot{\mathbf{x}}(t)=\mathbf{A}(t) \delta \mathbf{x}(t)+\mathbf{b}(t) u(t)+\mathbf{d}(\delta \mathbf{x}(t), \gamma(t), u(t)), \\
\mathbf{A}(t)=\partial \mathbf{f}(\mathbf{x}, 0) /\left.\partial \mathbf{x}\right|_{\mathbf{x}=\gamma(t)}, \mathbf{b}(t)=\partial \mathbf{f}(\gamma(t), u) /\left.\partial u\right|_{u=0}
\end{gathered}
$$

has $n-1$ multipliers strictly inside the unit cycle and one multiplier equals to one [AWK, YS], where $\delta \mathbf{x}(t)=\mathbf{x}(t)-\gamma(t)$, the matrix function $\mathbf{A}$ and the vector function $\mathbf{b}$ are $T$-periodic due to properties of $\gamma$. The function $\mathbf{d}(\delta \mathbf{x}(t), \gamma(t), u(t))$ represents the high order terms with respect to $\delta \mathbf{x}(t)$ in the system (4) linearization and for all $\mathbf{x} \in \mathcal{A}$ and $|u| \leq U$ there exist $d_{1}>0, d_{2}>0$ such that (the function $\mathbf{d}$ contains products of $\delta \mathbf{x}$ and $u$ with power 2 and higher):

$$
|\mathbf{d}(\delta \mathbf{x}, \gamma, u)| \leq d_{1}|\delta \mathbf{x}|^{2}+d_{2} u^{2}
$$

Multipliers are the eigenvalues of the monodromy matrix $\mathbf{M}=\boldsymbol{\Phi}(T)$ defined via the fundamental matrix function $\mathbf{\Phi}$ of the system (5) and the solution of adjoint system $\mathbf{\Psi}$ :

$$
\dot{\boldsymbol{\Phi}}(t)=\mathbf{A}(t) \boldsymbol{\Phi}(t), \boldsymbol{\Phi}(0)=\mathbf{I} ; \dot{\mathbf{\Psi}}(t)=-\mathbf{A}(t)^{T} \boldsymbol{\Psi}(t), \boldsymbol{\Psi}(0)=\mathbf{I},
$$

I is the identity matrix, $\boldsymbol{\Phi}(t)^{T} \boldsymbol{\Psi}(t)=\mathbf{I}$.

\section{B. Phase variables}

Any point $\mathbf{x}_{0} \in \Gamma$ can be characterized by a scalar phase $\varphi_{0} \in[0,2 \pi)$, which uniquely determines the position of the point $\mathbf{x}_{0}$ on the limit cycle $\Gamma$, because the limit cycle is a one-dimensional closed curve in $R^{n}$ [B, I, PRK]. The smooth bijective phase map $\vartheta: \Gamma \rightarrow[0,2 \pi)$ assigns to each point $\mathbf{x}_{0} \in \Gamma$ the corresponding phase $\varphi_{0}=\vartheta\left(\mathbf{x}_{0}\right)$. Any solution of the system (4) on the cycle $\mathbf{x}\left(t, \mathbf{x}_{0}, 0\right)$ (the system (4) solution with the initial condition $\mathbf{x}_{0}$ and input $u$ is denoted as $\left.\mathbf{x}\left(t, \mathbf{x}_{0}, u\right)\right), \mathbf{x}_{0} \in \Gamma$ satisfies $\mathbf{x}\left(t, \mathbf{x}_{0}, 0\right)=\gamma\left(t+\varphi_{0} \omega^{-1}\right), \omega=2 \pi / T$, provided we choose the convention $\gamma(t)=\mathbf{x}\left(t, \vartheta^{-1}(0), 0\right)$, then $\vartheta^{-1}(\varphi)=\gamma\left(\varphi \omega^{-1}\right)$. The phase variable $\varphi: R_{+} \rightarrow[0,2 \pi)$ is defined for the trajectories $\mathbf{x}\left(t, \mathbf{x}_{0}, 0\right), \mathbf{x}_{0} \in \Gamma$ as $\varphi(t)=\vartheta\left[\mathbf{x}\left(t, \mathbf{x}_{0}, 0\right)\right]=\Im\left[\gamma\left(t+\varphi_{0} \omega^{-1}\right)\right]$. Due to periodic nature of $\gamma(t)$, the function $\varphi(t)$ is also periodic, moreover the function $\vartheta$ can be defined in the particular way providing $\varphi(t)=\omega t+\varphi_{0}, \dot{\varphi}(t)=\omega[\mathrm{I}$, PRK].

The notion of phase can be extended to any solution $\mathbf{x}\left(t, \mathbf{x}_{0}, 0\right)$ starting in the attracted set $\mathcal{A}$. By definition of the attraction, for all $\mathbf{x}_{0} \in \mathcal{A}$ there exists an asymptotic phase $\theta_{0} \in[0,2 \pi)$ such that

$$
\lim _{t \rightarrow+\infty}\left|\mathbf{x}\left(t, \mathbf{x}_{0}, 0\right)-\gamma\left(t+\theta_{0} \omega^{-1}\right)\right|=0
$$

Then there exists the asymptotic phase map $\mathrm{v}: \mathcal{A} \rightarrow[0,2 \pi)$ connecting a point $\mathbf{x}_{0} \in \mathcal{A}$ and the corresponding phase $\theta_{0}$, i.e. $\theta_{0}=v\left(\mathbf{x}_{0}\right)$. The asymptotic phase variable $\theta: R_{+} \rightarrow[0,2 \pi)$ is derived as $\theta(t)=v\left[\mathbf{x}\left(t, \mathbf{x}_{0}, 0\right)\right], t \geq 0$ (it is 
supposed that $\mathbf{x}\left(t, \mathbf{x}_{0}, 0\right) \in \mathcal{A}$ for all $\left.t \geq 0\right)$. In the case $\varphi(t)=\omega t+\varphi_{0}$ we have $\theta(t)=\omega t+\theta_{0}$ and $\dot{\theta}(t)=\omega$, which implies invariance of this map: if $\mathrm{v}\left(\mathbf{x}_{1}\right)=\mathrm{v}\left(\mathbf{x}_{2}\right)$, then $\mathrm{v}\left[\mathbf{x}\left(t, \mathbf{x}_{1}, 0\right)\right]=\mathrm{v}\left[\mathbf{x}\left(t, \mathbf{x}_{2}, 0\right)\right]$ for all $t \geq 0$ and $\mathbf{x}_{1}, \mathbf{x}_{2} \in \mathcal{A}[\mathrm{I}]$. The initial conditions $\mathbf{x}_{1}, \mathbf{x}_{2} \in \mathcal{A}$ having the same asymptotical phase determine the isochrone curves [I]. Locally around $\Gamma$ the map $v$ coincides with the smooth map $\vartheta$ for all $\mathbf{x} \in \Gamma$.

The notion of asymptotic phase variable can be extended to a generic $u(t) \neq 0, t \geq 0$ provided that the corresponding trajectory $\mathbf{x}\left(t, \mathbf{x}_{0}, u\right)$ stays in the set $\mathcal{A}$ for all $t \geq 0$. In this case the asymptotic phase variable can be defined in a trivial way as $\theta(t)=v\left[\mathbf{x}\left(t, \mathbf{x}_{0}, u\right)\right], t \geq 0$. Then the variable $\theta\left(t^{\prime}\right)$ at an instant $t^{\prime} \geq 0$ evaluates the asymptotic phase of the point $\mathbf{x}\left(t^{\prime}, \mathbf{x}_{0}, u\right)$ if one would pose $u(t)=0$ for $t \geq t^{\prime}$. Dynamics of the asymptotic phase variable $\theta(t)$ in the generic case for $u(t) \neq 0, t \geq 0$ is hard to derive. A local model obtained in a small neighborhood of the limit cycle for infinitesimal inputs is presented below [I, PRK].

\section{Infinitesimal PRC}

Consider the case $u(t)=0$ for $t \geq 0$, then by definition $\dot{\gamma}(t)=\mathbf{f}(\gamma(t), 0), \quad \ddot{\gamma}(t)=\mathbf{A}(t) \dot{\gamma}(t)$ and $\dot{\gamma}(t)=\boldsymbol{\Phi}(t) \dot{\gamma}(0)$ for all $t \geq 0$. Therefore, $\dot{\gamma}(0)=\mathbf{f}(\gamma(0), 0)$ is the left eigenvector of the matrix $\mathbf{M}$ for the eigenvalue $\lambda_{1}(\mathbf{M})=1$. There exists right a eigenvector $\mathbf{m} \in R^{n}$ such that $\mathbf{m}^{T} \mathbf{M}=\mathbf{m}^{T}$ and $\mathbf{m}^{T} \dot{\gamma}(0)=\omega$. Finally, define $\mathbf{Q}(t)=\mathbf{m}^{T} \boldsymbol{\Psi}(t)^{T}$ then

$$
\mathbf{Q}(t) \mathbf{f}(\gamma(t), 0)=\mathbf{m}^{T} \boldsymbol{\Psi}(t)^{T} \mathbf{f}(\gamma(t), 0)=\mathbf{m}^{T} \boldsymbol{\Psi}(t)^{T} \boldsymbol{\Phi}(t) \dot{\gamma}(0)=\mathbf{m}^{T} \dot{\gamma}(0)=\omega .
$$

From another side, $\theta(t)=v[\gamma(t)]=\omega t+\theta(0)$ and

$$
\omega=\dot{\theta}=\partial v(\mathbf{x}) /\left.\partial \mathbf{x}\right|_{\mathbf{x}=\gamma(t)} \mathbf{f}(\gamma(t), 0) .
$$

Therefore $\mathbf{Q}(t)=\partial v(\mathbf{x}) /\left.\partial \mathbf{x}\right|_{\mathbf{x}=\gamma(t)}+\zeta(t)$, where $\zeta(t)$ is a row-vector orthogonal to $\mathbf{f}(\gamma(t), 0)$ (as an example $\zeta(t)=\tilde{\mathbf{m}}_{i}^{T} \boldsymbol{\Psi}(t)$ for some right eigenvector $\tilde{\mathbf{m}}_{i} \in R^{n}$ such that $\tilde{\mathbf{m}}_{i}^{T} \mathbf{M}=\lambda_{i}(\mathbf{M}) \tilde{\mathbf{m}}_{i}^{T}$ for the eigenvalue $\lambda_{i}(\mathbf{M}) \neq 1$, $i=2, \ldots, n)$. The map $\partial v(\mathbf{x}) /\left.\partial \mathbf{x}\right|_{\mathbf{x}=\gamma(t)}$ is independent on perturbations orthogonal to the limit cycle flow $\mathbf{f}(\gamma(t), 0)$ (only shifts in the direction of the limit cycle are tabulated). Since $\mathbf{m}$ is the eigenvector corresponding to movement on the limit cycle, then by the same reason $\mathbf{Q}(t)=\mathbf{m}^{T} \boldsymbol{\Psi}(t)^{T}$ is also independent of perturbations orthogonal to the limit cycle flow $\mathbf{f}(\gamma(t), 0)$. Therefore the convention $\mathbf{Q}(t)=\partial \mathbf{v}(\mathbf{x}) /\left.\partial \mathbf{x}\right|_{\mathbf{x}=\gamma(t)}=\mathbf{m}^{T} \boldsymbol{\Psi}(t)^{T}$ is adopted. The first equality explains the physical meaning of $\mathbf{Q}(t)$, while the last equality used for numerical calculation. The function $\mathbf{Q}(t)$ is $T$ periodic by construction.

The function $\mathbf{Q}\left(\varphi \omega^{-1}\right)$ for phase $\varphi \in[0,2 \pi)$ is called infinitesimal PRC [I]. Infinitesimal PRC $\mathbf{Q}$ serves as the phase response characteristics for a delta-impulse input $[\mathrm{I}]$.

\section{Phase dynamics}

Consider the case $u(t) \neq 0$ (we assume that $\mathbf{x}\left(t, \mathbf{x}_{0}, u\right) \in \mathcal{A}$ for all $t \geq 0$ ), then

$$
\begin{aligned}
& \dot{\theta}(\mathbf{x}(t))=\dot{\theta}(\gamma(t)+\delta \mathbf{x}(t))=\partial v(\mathbf{x}) /\left.\partial \mathbf{x}\right|_{\mathbf{x}=\gamma(t)+\delta \mathbf{x}(t)} \mathbf{f}(\gamma(t)+\delta \mathbf{x}(t), u(t))= \\
& =\partial v(\mathbf{x}) /\left.\partial \mathbf{x}\right|_{\mathbf{x}=\gamma(t)} \mathbf{f}(\gamma(t), u(t))+\mathbf{r}_{1}(\gamma(t), \delta \mathbf{x}(t), u(t))^{T} \delta \mathbf{x}(t),
\end{aligned}
$$


where the term $\mathbf{r}_{1}(\gamma(t), \delta \mathbf{x}(t), u(t))^{T} \delta \mathbf{x}(t)$ corresponds to the powers of $\delta \mathbf{x}(t)$ higher than one in the Taylor's series of the function $\dot{\theta}(\gamma(t)+\delta \mathbf{x}(t))$ with respect to the variable $\delta \mathbf{x}(t)$. From above, the quantity $\dot{\theta}(t)=\omega$ should be satisfied for $u(t)=0$, therefore $\partial v(\mathbf{x}) /\left.\partial \mathbf{x}\right|_{\mathbf{x}=\gamma(t)} \mathbf{f}(\gamma(t), 0)+\mathbf{r}_{1}(\gamma(t), \delta \mathbf{x}(t), 0)^{T} \delta \mathbf{x}(t)=\omega$, which implies the property $\mathbf{r}_{1}(\gamma(t), \delta \mathbf{x}(t), 0)=0$. Next,

$$
\begin{gathered}
\dot{\theta}(\mathbf{x}(t))=\partial v(\mathbf{x}) /\left.\partial \mathbf{x}\right|_{\mathbf{x}=\gamma(t)} \mathbf{f}(\gamma(t), 0)+\partial v(\mathbf{x}) /\left.\partial \mathbf{x}\right|_{\mathbf{x}=\gamma(t)} \partial \mathbf{f}(\gamma(t), u(t)) /\left.\partial u\right|_{u=0} u+g(\gamma(t), \delta \mathbf{x}(t), u(t)), \\
g(\gamma(t), \delta \mathbf{x}(t), u(t))=\mathbf{r}_{1}(\gamma(t), \delta \mathbf{x}(t), u(t))^{T} \delta \mathbf{x}(t)+r_{2}(\gamma(t), u(t)) u(t)^{2}
\end{gathered}
$$

where $r_{2}(\gamma(t), u(t)) u^{2}$ represents the terms with powers two and higher for the Taylor's series of the function $\partial v(\mathbf{x}) /\left.\partial \mathbf{x}\right|_{\mathbf{x}=\gamma(t)} \mathbf{f}(\gamma(t), u(t))$ with respect to the control $u$. For all $\mathbf{x} \in \mathcal{A}$ and $|u| \leq U$ there are $g_{1}>0$ and $g_{2}>0$ such that $|g(\gamma, \delta \mathbf{x}, u)| \leq g_{1} u^{2}+g_{2}|u \| \delta \mathbf{x}|$. Recalling the previously introduced designations we obtain

$$
\dot{\theta}=\omega+\mathbf{Q}(t) \mathbf{b}(t) u(t)+g(\gamma(t), \delta \mathbf{x}(t), u(t)) .
$$

This model has been derived around solution $\gamma(t)$, due to periodicity of the solution $\gamma\left(t+\varphi \omega^{-1}\right), \varphi \in[0,2 \pi)$ and $u$, the model for $\gamma\left(t+\varphi \omega^{-1}\right)$ has a similar form [I, K, W]:

$$
\dot{\theta}=\omega+\mathbf{Q}\left(t+\varphi \omega^{-1}\right) \mathbf{b}\left(t+\varphi \omega^{-1}\right) u(t)+g\left(\gamma\left(t+\varphi \omega^{-1}\right), \delta \mathbf{x}(t), u(t)\right) .
$$

\section{PRC-based control design for a single oscillator}

This section starts with the introduction of an approximated PRC-based model. Next, for the reduced PRC model two control algorithms are designed.

\section{A. Approximated PRC-based model}

Skipping the residual function $g$ we obtain the first order approximation of the model (6):

$$
\dot{\theta}=\omega+\mathbf{Q}\left(t+\varphi \omega^{-1}\right) \mathbf{b}\left(t+\varphi \omega^{-1}\right) u(t) .
$$

Since the property $|g(\gamma, \delta \mathbf{x}, u)| \leq g_{1} u^{2}+g_{2}|u \| \delta \mathbf{x}|$ holds for all $\mathbf{x} \in \mathcal{A}$ and $|u| \leq U$, such an approximation is rather accurate for the inputs $u$ with sufficiently small amplitude. Indeed, if $U \leq \varepsilon$ and $\varepsilon>0$ is chosen small enough, then deviations of trajectories from the limit cycle possess the estimate $|\delta \mathbf{x}| \leq \alpha \varepsilon$ for some $\alpha>0$, which gives the inequality $|g(\gamma, \delta \mathbf{x}, u)| \leq\left(g_{1}+g_{2} \alpha\right) \varepsilon^{2}$ and the first order terms in (6) dominate.

Let $\theta_{d}(t)=\omega t$ be the reference for the variable $\theta$, then $\chi(t)=\theta(t)-\theta_{d}(t) \in[0,2 \pi)$ is the resetting error and

$$
\dot{\chi}=\mathbf{Q}\left(t+\varphi \omega^{-1}\right) \mathbf{b}\left(t+\varphi \omega^{-1}\right) u(t) .
$$

Assume that the input $u(t)=w(t)$, where $w(t)$ has a pulse-like form, i.e. $|w(t)| \leq U$ for all $0<t<\mathcal{T}<T$ and $w(t)=0$ for all $t \geq \mathcal{T}$ or $t \leq 0$. Then integration of (7) yields for $t \geq \mathcal{T}$ (note that $\chi(0)=\theta(0)=\vartheta(\mathbf{x}(0)$ ) for all $\mathbf{x}(0) \in \Gamma)$ :

$$
\begin{aligned}
\chi(t) & =\chi(0)+\int_{0}^{t} \mathbf{Q}\left(\tau+\chi(0) \omega^{-1}\right) \mathbf{b}\left(\tau+\chi(0) \omega^{-1}\right) u(\tau) d \tau= \\
& =\chi(0)+\operatorname{PRC}(\chi(0)), \quad \operatorname{PRC}(\varphi)=\int_{0}^{\mathcal{T}} \mathbf{Q}\left(\tau+\varphi \omega^{-1}\right) \mathbf{b}\left(\tau+\varphi \omega^{-1}\right) w(\tau) d \tau .
\end{aligned}
$$

In (8) the map PRC is defined for the particular pulse $w$, such kind of PRC definition is rather common [GS, I, RSSM, 
TGPD], for the delta-impulse input and constant $\mathbf{b}$ it reduces to the infinitesimal PRC $\mathbf{Q}$ (by the definition $-\pi \leq \operatorname{PRC}(\varphi)<\pi$ for all $\varphi \in[0,2 \pi))$. The model (8) is a one step counterpart of the model (2) in the motivating example.

Let a train of pulses be given, then there exists a series of time instants $t_{i}, i \geq 1, t_{i+1}-t_{i} \geq T_{s}>\mathcal{T}$ such that the pulse is activated at time instants $t_{i}$ for all $i \geq 1$ :

$$
u(t)=\sum_{i=1}^{+\infty} w\left(t-t_{i}\right)
$$

The sampling constant $T_{s}$ depends on decaying properties of the trajectories after the pulse excitation. Denote $\chi_{i}=\chi\left(t_{i}\right)$ for $i \geq 1$. Since $u(t)=0$ for $0 \leq t \leq t_{1}$, then the error $\chi(t)$ is not changing on this time interval and $\theta(t)=\omega t+\theta(0)$. Therefore $\chi_{1}=\chi(0)=\theta(0)$ and the phase $\theta\left(t_{1}\right)=\omega t_{1}+\chi_{1}$. According to (8), after the first pulse

$$
\chi_{2}=\chi_{1}+\operatorname{PRC}\left(\omega t_{1}+\chi_{1}\right)
$$

and $\theta(t)=\omega t+\chi_{2}$ for $t_{1}+T_{s} \leq t \leq t_{2}$ because $u(t)=0$ for the duration. Therefore, $\theta\left(t_{2}\right)=\omega t_{2}+\chi_{2}$ and linearization of the system (4) dynamics has to be done around the new trajectory $\gamma\left(t+\theta\left(t_{2}\right) \omega^{-1}\right)$. Then according to (8), the new phase resetting error value after the second pulse equals

$$
\chi_{3}=\chi_{2}+\operatorname{PRC}\left(\omega t_{2}+\chi_{2}\right) \text {. }
$$

Again $\theta(t)=\omega t+\chi_{3}$ for $t \geq t_{2}+T_{s}$, the phase $\theta\left(t_{3}\right)=\omega t_{3}+\chi_{3}$ and from (8):

$$
\chi_{4}=\chi_{3}+\operatorname{PRC}\left(\omega t_{3}+\chi_{3}\right) \text {. }
$$

Repeating these calculations for each $i \geq 1$ we finally obtain:

$$
\chi_{i+1}=\chi_{i}+\operatorname{PRC}\left(\omega t_{i}+\chi_{i}\right)
$$

where we assume that all summation operations in the right hand side of (9) are done by modulo $2 \pi$. If $t_{i}=i T$, then the formula (9) is reduced to

$$
\chi_{i+1}=\chi_{i}+\operatorname{PRC}\left(\chi_{i}\right)
$$

that coincides with (2). The equation (9) describes the phase resetting evolution originated by a train of pulses under condition of the first approximation model validity for the system (4).

\section{B. PRC-based control design}

There exists one "free" parameter $t_{i}$ in the model (9), which is available for adjustment (the time instant when the next pulse $w$ is introduced). Assigning $t_{i}, i \geq 1$ one may ensure a desired phase resetting for the system (4). Let $\varpi_{i}=\theta\left(t_{i}\right)=\omega t_{i}+\chi_{i} \in[0,2 \pi), i \geq 1$ be the controlled phase of the pulse $w$ activation, then the model (9) can be rewritten in a form similar to $(3)$ :

$$
\chi_{i+1}=\chi_{i}+\operatorname{PRC}\left(\varpi_{i}\right), i \geq 1
$$

The problem is to design a sequence of $\varpi_{i}, i \geq 1$ providing the phase resetting from any initial phase error $\chi_{1} \in[0,2 \pi)$ to the zero. In this work as in [DM, ESS, E11] we assume that the PRC map has particular properties (it is similar to type II PRC from [HMM]). The corresponding control strategies for other types of PRC can be easily deduced from this main case.

Assumption 1. The map PRC is continuous and it has one zero $\varphi_{s}^{0} \in[0,2 \pi)$ with negative slope and another $\varphi_{u}^{0} \in[0,2 \pi)$ with positive slope, $\varphi_{s}^{0}<\varphi_{u}^{0}$. 
Since the map $P R C$ is $2 \pi$-periodic, then the zeros can be arranged in the required order $\varphi_{s}^{0}<\varphi_{u}^{0}$ changing the initial point on the limit cycle. Assumption 1 completely describes the form of PRC. Define

$$
\begin{gathered}
\varphi_{\max }=\arg \sup _{\varphi \in[0,2 \pi)} \operatorname{PRC}(\varphi), P R C_{\max }=P R C\left(\varphi_{\max }\right) ; \\
\varphi_{\min }=\arg \operatorname{anf}_{\varphi \in[0,2 \pi)} \operatorname{PRC}(\varphi), P R C_{\min }=\operatorname{PRC}\left(\varphi_{\min }\right),
\end{gathered}
$$

and $\varphi_{s}^{0}<\varphi_{\min }<\varphi_{u}^{0}<\varphi_{\max }, P R C_{\max }>0, P R C_{\min }<0$. Obviously, $\varphi_{s}^{0}$ corresponds to the stable equilibrium of the system (9) (for the periodic excitation, i.e., $t_{i}=i T, i \geq 1$ ) and $\varphi_{u}^{0}$ is the unstable one.

The model (10) is a first order discrete nonlinear system; such a class of systems is well investigated in the control theory literature [O] (that is an advantage of the model (10) compared it with (4)). In the work [ESS] two strategies for $\varpi_{i}$ design were proposed: feedforward and feedback control algorithms. A peculiarity of the phase resetting consists in the fact that $\chi \in[0,2 \pi)$, thus shift of the phase in both directions is possible for the resetting. To choose the direction one has to analyze what strategy (decreasing or increasing phase) leads to the fastest phase resetting. Define

$$
n_{\text {inc }}=\left(2 \pi-\chi_{1}\right) / P R C_{\max }, n_{d e c}=-\chi_{1} / P R C_{\min },
$$

where the integer parts of the numbers $n_{i n c}$ and $n_{d e c}$ determine the number of steps required for the initial phase $\chi_{1}$ resetting into a neighborhood of the zero applying increasing or decreasing strategy. These numbers are minimal since for their calculation we use the maximum reallocating amplitudes $P R C_{\max }$ and $P R C_{\min }$ achievable in both directions. Next, in this neighborhood the phase can be reset to the desired one applying one step shift with the same strategy due to assumed continuity of the map $P R C$. Therefore, the resetting requires $N+1$ pulses, $N=\operatorname{round}\left[\min \left\{n_{\text {inc }}, n_{d e c}\right\}\right]$, where the function $\operatorname{round}[n]$ returns the greatest integer not bigger than $n$. The following feedforward control has been proposed in [ESS] to solve the problem:

$$
\begin{gathered}
\varpi_{i}=\left\{\begin{array}{l}
\varphi_{\max } \text { if } n_{i n c} \leq n_{d e c} ; \quad 1 \leq i<N+1 ; \\
\varphi_{\min } \text { if } n_{i n c}>n_{d e c},
\end{array}\right. \\
\operatorname{PRC}\left(\varpi_{N+1}\right)+\chi_{N+1}=0, \\
\chi_{i+1}=\left\{\begin{array}{l}
\chi_{1}+P R C_{\max } i \text { if } n_{i n c} \leq n_{d e c} ; \quad 1 \leq i \leq N, \\
\chi_{1}+P R C_{\min } i \text { if } n_{i n c}>n_{d e c},
\end{array}\right.
\end{gathered}
$$

where the last step control $\varpi_{N+1}$ is calculated as a solution of the equation (12). In the control (11), (12) it is assumed that $\chi_{i}, i \geq 1$ are derived via (10) with the control (11) substitution (the formula (13)) and $\chi_{N+2}=0$ due to (12). This strategy has been called "feedforward" since it does not establish any relation with a real value of the phase.

Finally, the pulse activation instants $t_{i}, \quad 1 \leq i \leq N+1$ are calculated from (11), (12) tacking in mind that $t_{i+1}-t_{i} \geq T_{s}, 1 \leq i \leq N+1$ (the condition of the models (10) validity) [ESS]:

$$
t_{i+1}=t_{i}+s\left[\left(\varpi_{i+1}-\chi_{i+1}\right) \omega^{-1}\right], t_{0}=0,0 \leq i \leq N ; s(\tau)=\left\{\begin{array}{l}
\tau \text { if } \tau \geq T_{s} ; \\
\tau+T \text { otherwise. }
\end{array}\right.
$$

The feedback strategy assumes on-line measurements of the current phase after each pulse application, which increases the resetting accuracy. To realize this strategy, it is enough to replace the values $\chi_{i}$ in (11) and (12) generated by (13) with $\chi(t)$ available via measurements. For the phase resetting error, the term "measurements" means a calculation based on the state $\mathbf{x}(t)$. If a part of vector $\mathbf{x}(t)$ is available for measurements, then an observer can be applied to reconstruct the $\mathbf{x}(t)$ and the variable $\chi(t)$ next. The overall strategy for control design is similar to (11)-(13) [ESS]: 


$$
\begin{gathered}
n_{i n c}^{i}=\left(2 \pi-\chi_{i}\right) / P R C_{\max }, n_{d e c}^{i}=-\chi_{i} / P R C_{\min }, \\
\varpi_{i}=\left\{\begin{array}{l}
\varphi_{\max } \text { if } 1 \leq n_{i n c}^{i} \leq n_{d e c}^{i} ; \\
\varphi_{\min } \text { if } n_{i n c}^{i}>n_{d e c}^{i} \geq 1 ; i \geq 1 ; \\
\ell\left(\chi_{i}\right) \text { otherwise, }
\end{array}\right. \\
t_{i+1}=t_{i}+s\left[\left(\varpi_{i+1}-\chi_{i+1}\right) \omega^{-1}\right], t_{0}=0, i \geq 0,
\end{gathered}
$$

where the function $\ell(\chi)$ represents a solution of the equation $\operatorname{PRC}(\ell(\chi))+\chi=0$, the function $s$ is defined in (14). The feedback control strategy persists under convergent perturbations, which is an advantage with respect to the feedforward control. However, the feedback approach requires more measurement information and it has more computational complexity. Application of the feedforward strategy becomes more reliable if on-line measurements are not realizable or too noisy.

These strategies optimize the number of phase resetting steps and ensure the fastest phase resetting for (10). In the presence of uncertainties in the system model (4) (or inaccuracies of the first approximation) this optimality loses its advantages. In a such case the exponential asymptotic stabilization ensures a better performance due to its robustness. The exponentially stabilizing feedback for the model (10) is described by

$$
\operatorname{PRC}\left(\varpi_{i}\right)=-\kappa \chi_{i}, \kappa=\min \left\{-P R C_{\min }, P R C_{\max }\right\} / \pi,
$$

for all $i \geq 1$, where the time instant $t_{i}$ of pulse activation is calculated in accordance with (17) (in this case we assume that $\left.\chi_{i} \in[-\pi, \pi)\right)$. Under assumption 1 for the chosen $\kappa$ there always exists a value $\varpi_{i}$ such that $P R C\left(\varpi_{i}\right)=-\kappa \chi_{i}$. Substitution of (18) in the model (10) gives

$$
\chi_{i+1}=(1-\kappa) \chi_{i}
$$

which ensures an asymptotically exponential convergence of $\chi_{i}$ to zero since $0<\kappa<1$. Note that asymptotic stabilization implies that an infinite train of pulses has to be generated.

\section{Phase resetting for the model (4)}

In this section we are going to show that the control (17), (18) derived for the first order approximation model (10) is actually valid for the original nonlinear model (4) under some mild conditions. These conditions deal with accuracy of the first approximation for (4). The accuracy depends on amplitude of the input $u$, to analyze this relation more rigorously and to formulate the conditions, let

$$
u(t)=\varepsilon \sum_{i=1}^{+\infty} w\left(t-t_{i}\right),
$$

where $0<\varepsilon \leq 1$ is the control amplitude parameter and for $\varepsilon=1$ we recover the input considered previously. Since the model (10) is affine in the controls, for (19) it can be rewritten as follows

$$
\chi_{i+1}=\chi_{i}+\varepsilon \operatorname{PRC}\left(\varpi_{i}\right),
$$

where the map $\operatorname{PRC}(\varphi)$ has been defined in (8). To realize this algorithm we assume that the variables $\chi_{i}, i \geq 0$ are available for measurements. Since $\chi(t)=\chi\left(t_{i}+\mathcal{T}\right)$ for all $t \geq t_{i}+\mathcal{T}, i \geq 1$, then it is enough to measure the phase variable $\theta(t)$ once per pulse application at any time instant $t_{i}+\mathcal{T} \leq t \leq t_{i+1}, i \geq 1$ (by a spike detection for neuron oscillators, for example).

Theorem 1. Assume

(H1) the system (4) for $u(t) \equiv 0, t \geq 0$ has the locally asymptotically stable limit cycle $\Gamma$ with a bounded open set of 
attraction $\mathcal{A}$;

(H2) the "pulse" $w(t)$ is given $(w(t)=0$ for all $t \leq 0$ and $t \geq \mathcal{T},|w(t)| \leq U$ for $0<t<\mathcal{T}$ );

(H3) assumption 1 holds for the corresponding map $\operatorname{PRC}(\varphi)$.

Then for the system (4), for any $\delta>0$ there exists $0<\varepsilon \leq 1$ such that the control (19) with the time instants $t_{i}, i \geq 1$ $\left(t_{i+1}-t_{i} \geq T_{s}>\mathcal{T}\right.$ for all $\left.i \geq 1\right)$ calculated in accordance with (17), (18), ensures $\overline{\lim }_{t \rightarrow+\infty}|\chi(t)| \leq \delta$ for all $\mathbf{x}_{0} \in \Gamma$.

Pr o of. Local asymptotic stability of the set $\Gamma$ for the system (4) implies that for any initial conditions $\mathbf{x}_{0} \in \Gamma$ there exist $\bar{\varepsilon}>0$ and $c_{1}>0$ such that $\operatorname{dist}\left[\mathbf{x}\left(t, \mathbf{x}_{0}, u\right), \Gamma\right] \leq c_{1} \varepsilon$ for any $\varepsilon \leq \bar{\varepsilon}$ and all $t \geq 0$ (this claim follows from analysis of the system (5) for $|u(t)| \leq \varepsilon U$, the constant $c_{1}$ is computed for given $w(t)$ and $T_{s}$ ). The dynamics of the asymptotic phase $\theta$ for the system (4) is governed by the equation (6), then

$$
\dot{\chi}=\mathbf{Q}\left(t+\varphi \omega^{-1}\right) \mathbf{b}\left(t+\varphi \omega^{-1}\right) u(t)+g\left(\gamma\left(t+\varphi \omega^{-1}\right), \delta \mathbf{x}(t), u(t)\right),
$$

where $\delta \mathbf{x}(t)=\mathbf{x}\left(t, \mathbf{x}_{0}, u\right)-\gamma\left(t+\varphi \omega^{-1}\right)$ and $\gamma\left(t+\varphi \omega^{-1}\right)$ is the "base" trajectory (the trajectory around which the linearization has been performed). By construction, for all $\mathbf{x} \in \mathcal{A}$ and $|u| \leq \varepsilon U$ there exist $g_{1}>0, g_{2}>0$ such that

$$
|g(\gamma, \delta \mathbf{x}, u)| \leq \varepsilon^{2} g_{1} U^{2}+\varepsilon g_{2} U|\delta \mathbf{x}| .
$$

The values $\chi_{i+1}=\chi\left(t_{i+1}\right)=\chi\left(t_{i}+\mathcal{T}\right) \in[-\pi, \pi), i \geq 1$ are available for measurements $\left(\chi_{1}=\chi(0)\right.$ is given), then for $t \in\left(t_{i}, t_{i}+\mathcal{T}\right)$ the trajectory $\gamma\left(t+\chi_{i} \omega^{-1}\right)$ can be used as the "base" one $\left(u(t)=0\right.$ for all $\left.t \in\left[t_{i}+\mathcal{T}, t_{i+1}\right]\right)$. From stability of the limit cycle $\Gamma$ discussed above and continuity of the PRC map stated in assumption 1, there exists $c_{2}>0$ such that $\left|\delta \mathbf{x}_{i}(t)\right| \leq c_{2} \varepsilon$ for all $t \in\left(t_{i}, t_{i}+\mathcal{T}\right)$, where $\delta \mathbf{x}_{i}(t)=\mathbf{x}\left(t, \mathbf{x}_{0}, u\right)-\gamma\left(t+\chi_{i} \omega^{-1}\right)$. Therefore,

$$
\dot{\chi}=\varepsilon \mathbf{Q}\left(t+\chi_{i} \omega^{-1}\right) \mathbf{b}\left(t+\chi_{i} \omega^{-1}\right) w\left(t-t_{i}\right)+g\left(\gamma\left(t+\chi_{i} \omega^{-1}\right), \delta \mathbf{x}_{i}(t), \varepsilon w\left(t-t_{i}\right)\right)
$$

for all $t \in\left(t_{i}, t_{i}+\mathcal{T}\right)$ and integration of this equation gives $\left(\varpi_{i}=\omega t_{i}+\chi_{i}\right)$

$$
\begin{aligned}
& \chi_{i+1}=\chi_{i}+\int_{t_{i}}^{t_{i}+\mathcal{T}} \varepsilon \mathbf{Q}\left(t+\chi_{i} \omega^{-1}\right) \mathbf{b}\left(t+\chi_{i} \omega^{-1}\right) w\left(t-t_{i}\right)+g\left(\gamma\left(t+\chi_{i} \omega^{-1}\right), \delta \mathbf{x}_{i}(t), \varepsilon w\left(t-t_{i}\right)\right) d t= \\
& =\chi_{i}+\varepsilon P R C\left(\varpi_{i}\right)+\int_{t_{i}}^{t_{i}+\mathcal{T}} g\left(\gamma\left(t+\chi_{i} \omega^{-1}\right), \delta \mathbf{x}_{i}(t), \varepsilon w\left(t-t_{i}\right)\right) d t,
\end{aligned}
$$

where the map $\operatorname{PRC}(\varphi)$ has been defined in (8). From (18) $P R C\left(\varpi_{i}\right)=-\kappa \chi_{i}, 0<\kappa<1$, then

$$
\begin{aligned}
& \left|\chi_{i+1}\right| \leq(1-\varepsilon \kappa)\left|\chi_{i}\right|+\int_{t_{i}}^{t_{i}+\mathcal{T}}\left|g\left(\gamma\left(t+\chi_{i} \omega^{-1}\right), \delta \mathbf{x}_{i}(t), \varepsilon w\left(t-t_{i}\right)\right)\right| d t \leq \\
& \quad \leq(1-\varepsilon \kappa)\left|\chi_{i}\right|+\int_{t_{i}}^{t_{i}+\mathcal{T}} g_{1} \varepsilon^{2} U^{2}+g_{2} \varepsilon^{2} U c_{2} d t \leq(1-\varepsilon \kappa)\left|\chi_{i}\right|+\varepsilon^{2} U \mathcal{T}\left(g_{1} U+g_{2} c_{2}\right) .
\end{aligned}
$$

This inequality provides the following estimate

$$
\left|\chi_{i}\right| \leq(1-\varepsilon \kappa)^{i}\left|\chi_{0}\right|+\varepsilon^{2} U \mathcal{T}\left(g_{1} U+g_{2} c_{2}\right) \sum_{j=0}^{i-1}(1-\varepsilon \kappa)^{j}, \sum_{j=0}^{+\infty}(1-\varepsilon \kappa)^{j}=(\varepsilon \kappa)^{-1},
$$

which implies the theorem claim for $\delta=\varepsilon \kappa^{-1} U \mathcal{T}\left(g_{1} U+g_{2} c_{2}\right)$.

The result of theorem 1 means that the exponentially stabilizing feedback (17), (18), designed for the reduced model (10), being applied to (4), ensures practical phase resetting. According to theorem conditions, this approach can be applied to any smooth dynamical system (4), with a limit cycle and infinitesimal pulse input, provided that the corresponding PRC map suits the requirements of Assumption 1.

Accuracy of the resetting can be regulated by decreasing the control amplitude $\varepsilon$, which in general may slow down the rate of convergence. It is worth stressing that since (17), (18) assumes an infinite series of pulses (a pulse is applied 
at each period), then decreasing the pulse amplitude causes the time of resetting to increase with simultaneous decrease of the energy. The trade-off between robustness of the system and the rate of convergence may consist in initial application of the suboptimal controls (11)-(14) or (15)-(17) with posterior activation of the feedback (17), (18). Indeed, the value of $\delta$ decreases if the feedback gain $\kappa$ is growing. From (18) the value of $\kappa$ is restricted by the amplitude of PRC and the admissible range of the phase resetting error, i.e. $|\chi| \leq \pi$. If we restrict the area where the control (18) is applied, say for $|\chi| \leq \varsigma \pi$ with $0<\varsigma<1$, when we can take

$$
\kappa=\min \left\{-P R C_{\min }, P R C_{\max }\right\} /(\varsigma \pi),
$$

that according to the result of theorem 1 implies improvement of the accuracy (decreasing of $\delta$ ).

Corollary 1 . Let conditions (H1)-(H3) of theorem 1 be satisfied. Take the control (19) with the time instants $t_{i}, i \geq 1\left(t_{i+1}-t_{i} \geq T_{s}>\mathcal{T}\right.$ for all $\left.i \geq 1\right)$ calculated in accordance with (17) and

$$
\varpi_{i}=\left\{\begin{array}{l}
\varphi_{\max } \text { if } 1 \leq n_{i n c}^{i} \leq n_{d e c}^{i} \\
\varphi_{\min } \text { if } n_{i n c}^{i}>n_{d e c}^{i} \geq 1 ; \quad i \geq 1 \\
\ell\left(\chi_{i}\right) \text { if }\left|\chi_{i}\right|>\varsigma \pi ; \\
\mu\left(\chi_{i}\right) \text { otherwise, }
\end{array}\right.
$$

where $n_{\text {inc }}^{i}, n_{\text {dec }}^{i}$ are from (15) and the function $\mu(\chi)$ represents a solution of the equation $P R C(\mu(\chi))+\kappa \chi=0$. Then for the system (4), for any $\delta>0$ there exists $0<\varepsilon \leq 1$ such that the control (17), (21), (22) ensures $\varlimsup_{t \rightarrow+\infty}|\chi(t)| \leq \delta$ for all $\mathbf{x}_{0} \in \Gamma$ provided that $\kappa<1$ in (21).

Proof. The proof mainly follows the ideas of the theorem. If $\left|\chi_{i}\right| \leq \varsigma \pi$, then the theorem arguments are valid directly. If $\left|\chi_{i}\right|>\varsigma \pi$, then according to (21) $\left|\chi_{i}+\varepsilon P R C\left(\varpi_{i}\right)\right| \leq(1-\varepsilon \kappa)\left|\chi_{i}\right|$ and again the proof follows the same line, since in both cases the inequality (20) is satisfied.

It is worth stressing that if $\chi_{i^{*}} \approx 0$ for some $i^{*} \geq 0$ (the resetting is successfully performed), then the algorithm (22) selects the controlling phase $\varpi_{i^{*}}$ such that $\operatorname{PRC}\left(\varpi_{i^{*}}\right)=0$. According to assumption 1 , in this case either $\varpi_{i^{*}} \approx \varphi_{s}^{0}$ or $\varpi_{i^{*}} \approx \varphi_{u}^{0}$. In order to improve the control robustness, it is desirable to realize the function $\mu$ such that the solution $\varpi_{i^{*}} \approx \varphi_{s}^{0}$ (or a point in its vicinity) is chosen in such situations. Indeed, since $\varpi_{i^{*}}=\omega t_{i^{*}}+\chi_{i^{*}} \approx \omega t_{i^{*}}$, then $t_{i^{*}} \approx \omega^{-1} \varphi_{s}^{0}$ and next from (17) we get $t_{i+1}=t_{i}+T$ for all $i \geq i^{*}$. Therefore, the control (22) generates a periodic train of pulses asymptotically. It acts as a conventional Poincaré phase map [I]. In the presence of uncertainties, if $\varpi_{i^{*}} \approx \varphi_{s}^{0}$, then this control will stabilize the phase deviations, while the solution $\varpi_{i^{*}} \approx \varphi_{u}^{0}$ may additionally perturb the system.

The advantages of this control technique will be demonstrated in Section 7.

\section{PRC-based control design for a network of oscillators}

In this section we will assume that a group of disconnected oscillators with a common input is given, i.e. $S>0$ is the number of oscillators and $\theta^{j}(t)$ is the phase of $j$-th oscillator, $j=1, \ldots S$. We will also assume that all oscillators are identical (they have a PRC map satisfying assumption 1) and that they have different initial phases $\varphi^{j} \in[0,2 \pi)$, $j=1, \ldots S$ (different initial conditions $\left.\mathbf{x}_{j}(0)\right)$. The desired phase $\theta_{d}(t)=\omega t$ is the common reference for all variables $\theta^{j}, j=1, \ldots S$. Introducing the phase resetting errors for the oscillators $\chi^{j}(t)=\theta^{j}(t)-\theta_{d}(t), j=1, \ldots S$, we obtain $\chi^{j}(0)=\varphi^{j}$. Then from (6) we get the equations for the phase dynamics of the population: 


$$
\dot{\chi}^{j}=\mathbf{Q}\left(t+\varphi^{j} \omega^{-1}\right) \mathbf{b}\left(t+\varphi^{j} \omega^{-1}\right) u(t)+g\left(\gamma\left(t+\varphi^{j} \omega^{-1}\right), \delta \mathbf{x}_{j}(t), u(t)\right), j=1, \ldots S
$$

where $\delta \mathbf{x}_{j}(t)=\mathbf{x}_{j}(t)-\gamma\left(t+\varphi^{j} \omega^{-1}\right)$ is the deviation of the trajectory of $j$-th oscillator from its basement on the limit cycle $\gamma\left(t+\varphi^{j} \omega^{-1}\right)$. We will assume that the average resetting error of the network

$$
y(t)=S^{-1} \sum_{j=1}^{S} \chi^{j}(t)
$$

is available for measurements (as before we will need this measurements only once per the pulse application). The common input is given in the form (19) as a train of pulses $w(t)$ with the adjustable instants of activation $t_{i}, i \geq 1$, where $\varepsilon>0$ is the parameter.

Since the initial phases $\varphi^{j}, j=1, \ldots S$ are not identical, then an application of the control approach developed in the previous sections may not lead to the synchronization and a desired resetting for all oscillators. The issue is that the phase model around limit cycle (23) has an integrator like form, thus it is marginally stable only. In such a case stability of linearized system does not necessary imply a similar property for the original nonlinear system. In the following, under a mild restriction on the PRC map and a modification of the control law, it is shown that it is not the case.

A s sumption 2 . Denote the set of values $\varphi \in[0,2 \pi)$ for which the map $\operatorname{PRC}(\varphi)$ is concave as $\hat{\Upsilon} \subset[0,2 \pi)$ and the set of values $\varphi \in[0,2 \pi)$ for which $\operatorname{PRC}(\varphi)$ is convex as $\breve{\Upsilon} \subset[0,2 \pi)$. Let there exist non empty open connected sets $\widehat{\Upsilon}_{-} \subset \widehat{\Upsilon}$ and $\breve{\Upsilon}_{+} \subset \breve{\Upsilon}$ such that

1) $\operatorname{PRC}(\varphi) \leq 0$ for all $\varphi \in \widehat{\Upsilon}_{-}$and $\operatorname{PRC}(\varphi) \geq 0$ for all $\varphi \in \breve{\Upsilon}_{+}$;

2) $\left\{\varphi_{s}^{0}, \varphi_{u}^{0}\right\} \subset \widehat{\Upsilon}_{-}$and $\left\{\varphi_{s}^{0}, \varphi_{u}^{0}\right\} \subset \breve{\Upsilon}_{+}$.

An example of the PRC map that satisfies assumption 2 is given in Fig. 8 for the Van de Pol model. This assumption regularizes the PRC map behavior (the shape of PRC can be adjusted by the pulse $w$ choice). Let $\varphi_{-} \in \widehat{\Upsilon}_{-}$and $\varphi_{+} \in \breve{\Upsilon}_{+}$be the median values of the sets $\widehat{\Upsilon}_{-}$and $\breve{\Upsilon}_{+}$respectively. Then the control algorithm can be formulated as follows:

$$
\begin{gathered}
n_{d e c}^{i}=\left|y_{i}\right| /\left|\operatorname{PRC}\left(\varphi_{-}\right)\right|, n_{i n c}^{i}=\left|y_{i}\right| /\left|\operatorname{PRC}\left(\varphi_{+}\right)\right|, \\
\varpi_{i}=\left\{\begin{array}{l}
\varphi_{-} \text {if } y_{i} \geq 0 \text { and } 1 \leq n_{d e c}^{i} \\
\varphi_{+} \text {if } y_{i}<0 \text { and } n_{i n c}^{i} \geq 1 ;, i \geq 1, \\
\ell\left(y_{i}\right) \text { otherwise, }
\end{array}\right. \\
t_{i+1}=t_{i}+s\left[\left(\varpi_{i+1}-y_{i+1}\right) \omega^{-1}\right], t_{0}=0, i \geq 0,
\end{gathered}
$$

where $y_{i}=y\left(t_{i}\right)=y\left(t_{i-1}+\mathcal{T}\right) \in[-\pi, \pi)$ and the functions $\ell, s$ have been defined of a previous section. Thus the control (24)-(26) does not repeat the structure of the control (17), (18) under substitution $y_{i}=\chi_{i}$ (this equality becomes true if the network consists in the single oscillator).

The or e m 2. Let conditions (H1), (H2) of theorem 1 and assumptions 1,2 be satisfied. Take the control (19) with the time instants $t_{i}, i \geq 1\left(t_{i+1}-t_{i} \geq T_{s}>\mathcal{T}\right.$ for all $\left.i \geq 1\right)$ calculated in accordance with (24)-(26). Then for the system (4) for any $\delta>0$ there exist $i^{*}>0,0<\varepsilon \leq 1$ and $\rho>0$ such that $\left|\chi^{j}\left(t_{i^{*}}\right)\right| \leq \delta$ for all $j=1, \ldots S$ provided that $\max _{j, k=1, \ldots, S}\left|\varphi^{j}-\varphi^{k}\right| \leq \rho$.

Pro of. Again, the local asymptotic stability of the set $\Gamma$ for the system (4) implies that for any initial conditions 
$\mathbf{x}_{0} \in \Gamma$ there exist $\bar{\varepsilon}>0$ and $c_{1}>0$ such that $\operatorname{dist}\left[\mathbf{x}\left(t, \mathbf{x}_{0}, u\right), \Gamma\right] \leq c_{1} \varepsilon$ for any $\varepsilon \leq \bar{\varepsilon}$ and all $t \geq 0$. By construction, for all $\mathbf{x} \in \mathcal{A}$ and $|u| \leq \varepsilon U$ there exist $g_{1}>0, g_{2}>0$ such that

$$
|g(\gamma, \delta \mathbf{x}, u)| \leq \varepsilon^{2} g_{1} U^{2}+\varepsilon g_{2} U|\delta \mathbf{x}|
$$

From (19) and (23) we get

$$
\dot{y}=\varepsilon S^{-1} \sum_{j=1}^{S}\left[\mathbf{Q}\left(t+\chi_{i}^{j} \omega^{-1}\right) \mathbf{b}\left(t+\chi_{i}^{j} \omega^{-1}\right) w\left(t-t_{i}\right)+g\left(\gamma\left(t+\chi_{i}^{j} \omega^{-1}\right), \delta \mathbf{x}_{i, j}(t), \varepsilon w\left(t-t_{i}\right)\right)\right]
$$

for all $t \in\left(t_{i}, t_{i}+\mathcal{T}\right)$, where $\delta \mathbf{x}_{i, j}(t)=\mathbf{x}_{j}(t)-\gamma\left(t+\chi_{i}^{j} \omega^{-1}\right)$. By the same arguments as in theorem 1 , there exists $c_{2}>0$ such that $\left|\delta \mathbf{x}_{i, j}(t)\right| \leq c_{2} \varepsilon$ for all $t \in\left(t_{i}, t_{i}+\mathcal{T}\right)$ for all $j=1, \ldots S$, then $|g(\gamma, \delta \mathbf{x}, u)| \leq \varepsilon^{2} U\left(g_{1} U+g_{2} c_{2}\right)$.

Consider the case $y_{0} \geq 0$. Integration on the time interval $\left[t_{i}, t_{i+1}\right], i \geq 1$ gives

$$
\begin{aligned}
& y_{i+1}=y_{i}+S^{-1} \sum_{j=1}^{S} \int_{t_{i}}^{t_{i}+\mathcal{T}} \varepsilon \mathbf{Q}\left(t+\chi_{i}^{j} \omega^{-1}\right) \mathbf{b}\left(t+\chi_{i}^{j} \omega^{-1}\right) w\left(t-t_{i}\right)+g\left(\gamma\left(t+\chi_{i}^{j} \omega^{-1}\right), \delta \mathbf{x}_{i, j}(t), \varepsilon w\left(t-t_{i}\right)\right) d t= \\
& =y_{i}+S^{-1} \sum_{j=1}^{S}\left[\varepsilon P R C\left(\omega t_{i}+\chi_{i}^{j}\right)+\int_{t_{i}}^{t_{i}+\mathcal{T}} g\left(\gamma\left(t+\chi_{i}^{j} \omega^{-1}\right), \delta \mathbf{x}_{i, j}(t), \varepsilon w\left(t-t_{i}\right)\right) d t\right],
\end{aligned}
$$

where the map $\operatorname{PRC}(\varphi)$ has been defined in (8). Note that

$$
\left|S^{-1} \sum_{j=1}^{S} \int_{t_{i}}^{t_{i}+\mathcal{T}} g\left(\gamma\left(t+\chi_{i}^{j} \omega^{-1}\right), \delta \mathbf{x}_{i, j}(t), \varepsilon w\left(t-t_{i}\right)\right) d t\right| \leq \varepsilon^{2} U \mathcal{T}\left(g_{1} U+g_{2} c_{2}\right)
$$

as in the single oscillator case, and by the Jensen's inequality

$$
S^{-1} \sum_{j=1}^{S} P R C\left(\omega t_{i}+\chi_{i}^{j}\right) \leq \operatorname{PRC}\left(S^{-1} \sum_{j=1}^{S} \omega t_{i}+\chi_{i}^{j}\right)=P R C\left(\omega t_{i}+y_{i}\right)
$$

provided that the PRC map is concave for all $\omega t_{i}+\chi_{i}^{j}, j=1, \ldots S$. Since $y_{1}=y_{0} \geq 0$ from (24)-(26) $\omega t_{1}+y_{1}=\varphi_{-} \in \widehat{\Upsilon}_{-} \subset \widehat{\Upsilon}$ and there exists $\rho>0$ such that $\omega t_{1}+\chi_{1}^{j} \in \widehat{\Upsilon}$, thus

$$
S^{-1} \sum_{j=1}^{S} \operatorname{PRC}\left(\omega t_{1}+\chi_{1}^{j}\right) \leq \operatorname{PRC}\left(S^{-1} \sum_{j=1}^{S} \omega t_{1}+\chi_{1}^{j}\right)=\operatorname{PRC}\left(\varphi_{-}\right)<0
$$

and

$$
\begin{aligned}
y_{2} & \leq y_{1}+S^{-1} \sum_{j=1}^{S} \varepsilon \operatorname{PRC}\left(\omega t_{1}+\chi_{1}^{j}\right)+\varepsilon^{2} U \mathcal{T}\left(g_{1} U+g_{2} c_{2}\right) \\
& \leq y_{1}+\varepsilon \operatorname{PRC}\left(\varphi_{-}\right)+\varepsilon^{2} U \mathcal{T}\left(g_{1} U+g_{2} c_{2}\right)
\end{aligned}
$$

Therefore, there exist an $\varepsilon>0$ such that $\varepsilon \operatorname{PRC}\left(\varphi_{-}\right)+\varepsilon^{2} U \mathcal{T}\left(g_{1} U+g_{2} c_{2}\right)=-\varepsilon \theta$ for some $\theta>0$. Next for any finite number of steps $i^{*}>0$ there is a sufficiently small $\rho>0$ such that $y_{i} \geq 0$ and $\omega t_{i}+\chi_{i}^{j} \in \widehat{\Upsilon}$ for all $i \geq 2$ due to introduced assumptions on PRC and the control law (24)-(26). Then

$$
y_{i+1} \leq y_{i}-\varepsilon \theta=y_{0}-i \varepsilon \theta
$$

and there exists a finite number $i^{*}>0$ such that $n_{i n c}^{i^{*}}<1$ and $y_{i^{*}+1} \leq \varepsilon^{2} U \mathrm{~T}\left(g_{1} U+g_{2} c_{2}\right)$. The case $y_{0} \leq 0$ can be treated similarly using the Jensen's inequality for convex functions.

The most restrictive condition of this theorem deals with a priori assumption that $\max _{j, k=1, \ldots, S}\left|\varphi^{j}-\varphi^{k}\right| \leq \rho$, which is hard to verify since the variables $\varphi^{j}, j=1, \ldots S$ are not measurable. However, applying a periodic train of pulses (19) with

$$
\varpi_{k}=\omega t_{k}+y_{k}=\varphi_{s}^{0}, k=1, \ldots, K
$$

where $K>0$ is the number of periods, we can ensure the convergence of the phases $\chi_{k}^{j}, j=1, \ldots S$ to $y_{k}$ guaranteeing that the condition $\max _{j, k=1, \ldots, S}\left|\varphi^{j}-\varphi^{k}\right| \leq \rho$ is satisfied for any $\rho>0$ for a sufficiently large $K$. 


\section{Applications}

For all examples we will consider the square pulses of the form:

$$
w(t)=\left\{\begin{array}{l}
\Delta \text { if } t>0 \wedge t<\delta ; \\
0 \text { otherwise }
\end{array}\right.
$$

where the values of parameters $\delta>0, \Delta>0$ will be specified later depending on the application.

\section{A. Motivating example}

Let $\delta=1$ and $\Delta=0.3$; the corresponding phase response curve (8) is plotted in Fig. 2. Assumption 1 is satisfied for this PRC map. The model (1) trajectory with the control (17), (18) is shown in Fig. 3. The symbol $\chi_{0}$ denotes the initial error, the symbol $\chi_{d}$ is stated for the desired error value. As we can conclude from this result the convergence rate is very slow in such system. To improve the rate of convergence it is proposed to combine the control (15)-(17) for the big resetting error and the control (17), (18) for asymptotical resetting. The corresponding trajectory with the control (17), (21), (22) for $\varsigma=0.1$ is plotted in Fig. 4. The time axis of figures 3 and 4 is scaled in the number of periods.

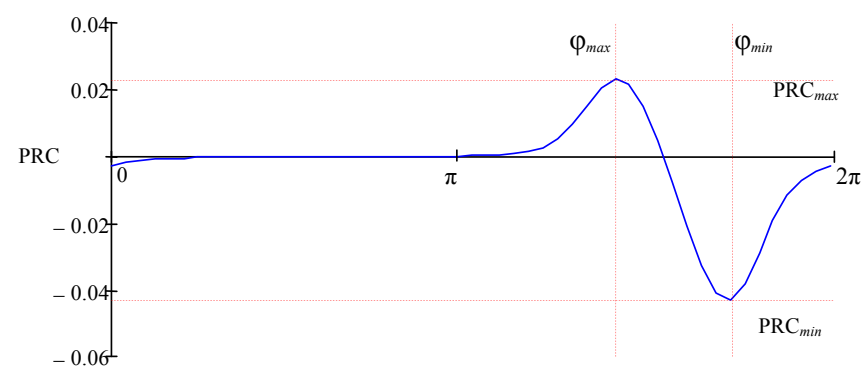

Fig. 2. PRC for the system (1).

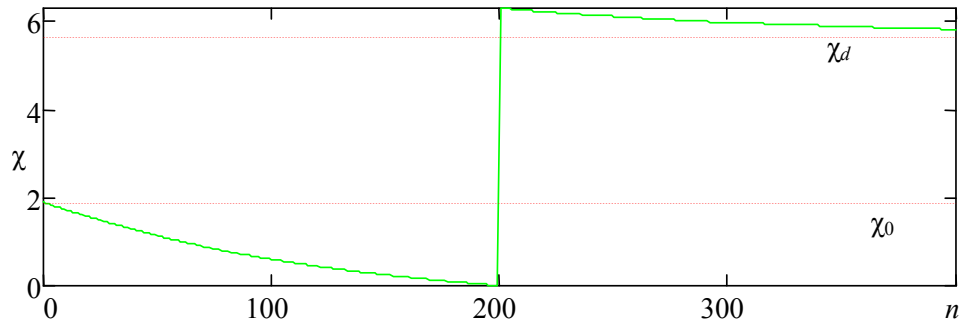

Fig. 3. The system (1) trajectory with the control (17), (18).

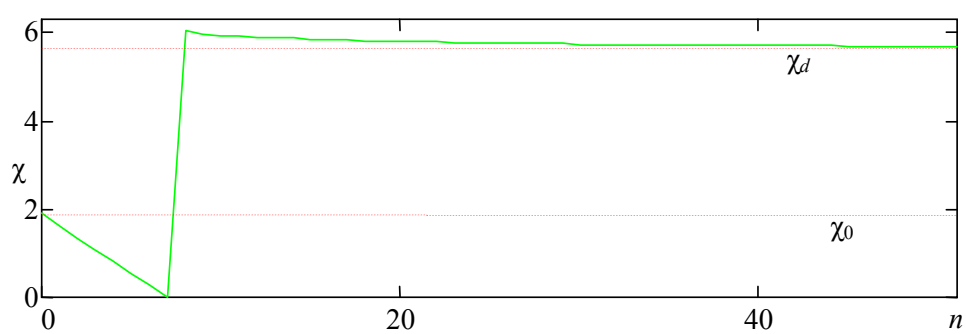

Fig. 4. The system (1) trajectory with the combined control (17), (21), (22).

\section{B. Tenth order model of circadian oscillations in Drosophila}

Let us consider the model from the paper [LG98, LG99]:

$$
\dot{M}_{P}=\left[v_{s p}+u(t)\right] \frac{K_{I P}^{n}}{K_{I P}^{n}+C_{N}^{n}}-v_{m P} \frac{M_{P}}{K_{m P}+M_{P}}-k_{d} M_{P} ;
$$




$$
\begin{gathered}
\dot{P}_{0}=k_{S P} M_{P}-V_{1 P} \frac{P_{0}}{K_{1 P}+P_{0}}+V_{2 P} \frac{P_{1}}{K_{2 P}+P_{1}}-k_{d} P_{0} ; \\
\dot{P}_{1}=V_{1 P} \frac{P_{0}}{K_{1 P}+P_{0}}-V_{2 P} \frac{P_{1}}{K_{2 P}+P_{1}}-V_{3 P} \frac{P_{1}}{K_{3 P}+P_{1}}+V_{4 P} \frac{P_{2}}{K_{4 P}+P_{2}}-k_{d} P_{1} ; \\
\dot{P}_{2}=V_{3 P} \frac{P_{1}}{K_{3 P}+P_{1}}-V_{4 P} \frac{P_{2}}{K_{4 P}+P_{2}}-k_{3} P_{2} T_{2}+k_{4} C-v_{d P} \frac{P_{2}}{K_{d P}+P_{2}}-k_{d} P_{2} ; \\
\dot{M}_{T}=v_{s p} \frac{K_{I T}^{n}}{K_{I T}^{n}+C_{N}^{n}}-v_{m T} \frac{M_{T}}{K_{m T}+M_{T}}-k_{d} M_{T} ; \\
\dot{T}_{1}=V_{1 T} \frac{T_{0}}{K_{1 T}+T_{0}}-V_{2 T} \frac{T_{1}}{K_{2 T}+T_{1}}-V_{3 T} \frac{T_{1}}{K_{3 T}+T_{1}}+V_{4 T} \frac{T_{2}}{K_{4 T}+T_{2}}-k_{d} T_{1} ; \\
\dot{T}_{2}=V_{3 T} \frac{T_{1}}{K_{3 T}+T_{1}}-V_{4 T} \frac{T_{2}}{K_{4 T}+T_{2}}-k_{3} P_{2} T_{2}+k_{4} C-v_{d T} \frac{T_{2}}{K_{d T}+T_{2}}-k_{d} T_{2} ; \\
\dot{C}=k_{3} P_{2} T_{2}-k_{4} C-k_{1} C+k_{2} C_{N}-k_{d C} C \\
\dot{C}_{N}=k_{1} C-k_{2} C_{N}-k_{d N} C_{N},
\end{gathered}
$$

where $M_{P}$ is cytosolic concentration of per mRNA; $P_{0}, P_{1}, P_{2}$ are unphosphorylated, monophosphorylated and bisphosphorylated concentrations of PER protein respectively; $M_{T}$ is cytosolic concentration of tim mRNA; $T_{0}, T_{1}$, $T_{2}$ are unphosphorylated, monophosphorylated and bisphosphorylated concentrations of TIM protein respectively; $C$ is PER-TIM complex concentration and $C_{N}$ is nuclear form of PER-TIM complex. As in [LG98] we will consider the following values of the model (27)-(36) parameters:

$$
\begin{gathered}
K_{I P}=K_{I T}=v_{s T}=v_{S P}=1, v_{m P}=v_{m T}=0.7, K_{d P}=K_{d T}=K_{m P}=K_{m T}=0.2, k_{S P}=k_{s T}=0.9, v_{d P}=v_{d T}=2, \\
K_{1 P}=K_{1 T}=K_{2 P}=K_{2 T}=K_{3 P}=K_{3 T}=K_{4 P}=K_{4 T}=2, V_{1 P}=V_{1 T}=V_{3 P}=V_{3 T}=8, \\
k_{1}=0.6, k_{2}=0.2, k_{3}=1.2, k_{4}=0.6, n=4, k_{d}=k_{d C}=k_{d N}=0.01, V_{2 P}=V_{2 T}=V_{4 P}=V_{4 T}=1 .
\end{gathered}
$$

The phase resetting for this model was considered in the papers [BSD1, BSD2] via optimal control approach. For the case $u=0$ this model has the single equilibrium and one stable limit cycle, $T=24.13$.

Let $\delta=1$ and $\Delta=0.3$; the corresponding phase response curve is plotted in Fig. 5. The assumption 1 is satisfied for the PRC map. The trajectory of the model (27)-(36) with the control (17), (18) is shown in Fig. 6, the trajectory with the control (17), (21), (22) for $\varsigma=0.1$ is plotted in Fig. 7. The time axis is scaled in the number of periods. The symbols $\chi_{0}$ and $\chi_{d}$ denote the initial and the desired error values.

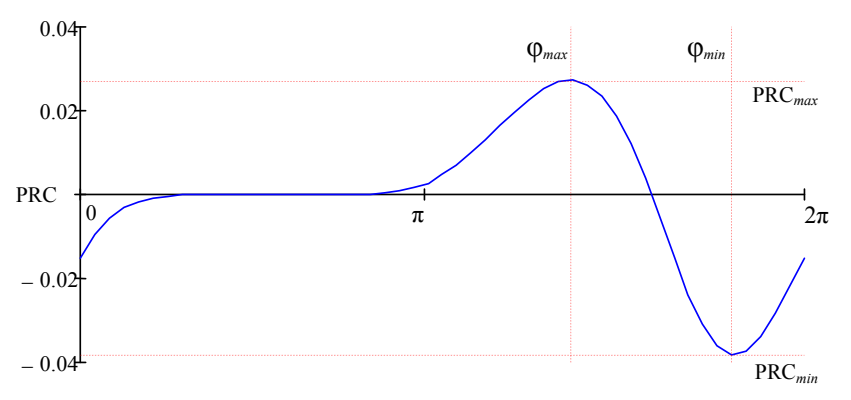

Fig. 5. PRC for the system (27)-(36). 


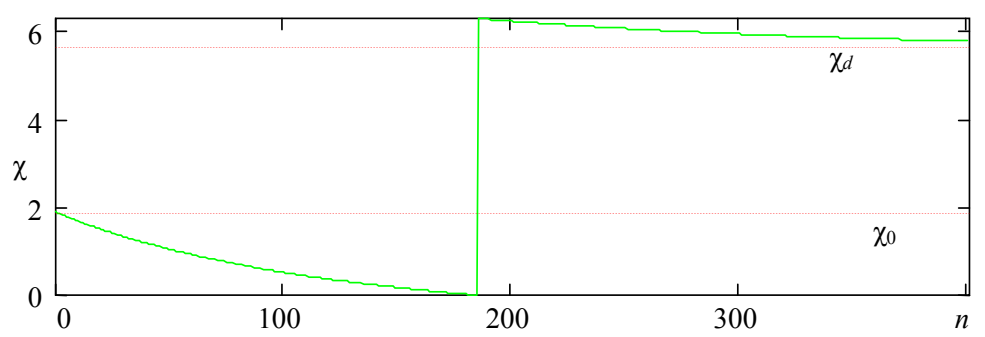

Fig. 6. The system (27)-(36) trajectory with the control (17), (18).

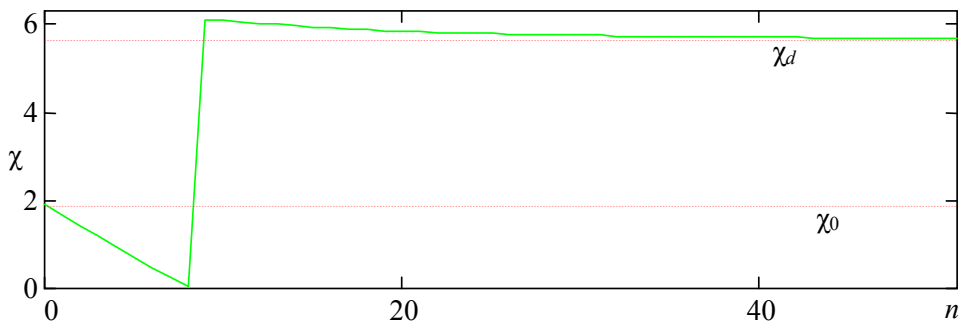

Fig. 7. The system (27)-(36) trajectory with the combined control (17), (21), (22).

The demonstrated rate of the resetting error convergence is rather slow, that is originated by pulses application with rather small amplitude. Such restriction comes from analytical conditions dealt with analysis of the first approximation arguments used for resetting.

\section{A network of Van der Pol oscillators}

In the works [Z, AFFS] a mammalian circadian pacemaker has been represented by a population of neurons modeled by Van der Pol differential equations:

$$
\begin{aligned}
\dot{x}_{j} & =\omega\left[y_{j}+\mu\left(x_{j}-v x_{j}^{3}\right)\right]+u, \\
\dot{y}_{j} & =-\omega x_{j},
\end{aligned}
$$

where $x_{j}, y_{j}$ are the neuron normalized coordinates, $j=1, \ldots, S ; S>0$ is the number of neurons in the network, $\omega>0$ is the neuron oscillation frequency, $\mu>0$ and $v>0$ are the model parameters; $u$ is the common input. In this work for all neurons we take as in [Z]

$$
\mu=0.2, \omega=0.262, v=1,
$$

then the neurons have a globally asymptotically stable limit cycle with $T=24.06$. Let $\delta=3$ and $\Delta=0.125$; the corresponding phase response curve is plotted in Fig. 8. The assumption 1 is satisfied for the PRC map, the sets $\hat{\Upsilon}_{-}$, $\breve{\Upsilon}_{+}$from assumption 2 are also shown in Fig. 8 (in these sets the PRC map can be rather accurately approximated by a straight line). The trajectories of a network for $S=10$ with the control (24)-(26) are shown in Fig. 9, the trajectories for the control (24)-(26) and an initial periodical train of pulses with $K=10$ (applied before the resetting) are plotted in Fig. 10. The time axis is scaled in the number of periods. The symbols $y_{0}$ and $y_{d}$ denote the initial and the desired error values for the averaged phase error. In this case the initial deviations of phases $\varphi^{j}$ in the network from $y_{0}$ have been chosen large enough, as we can conclude even in this case the control ensures the resetting (Fig. 9). The quality of resetting becomes much better in Fig. 10, where a periodic train of pulses is applied (in this case the phase deviations at the instant of the control (24)-(26) activation becomes much smaller). 


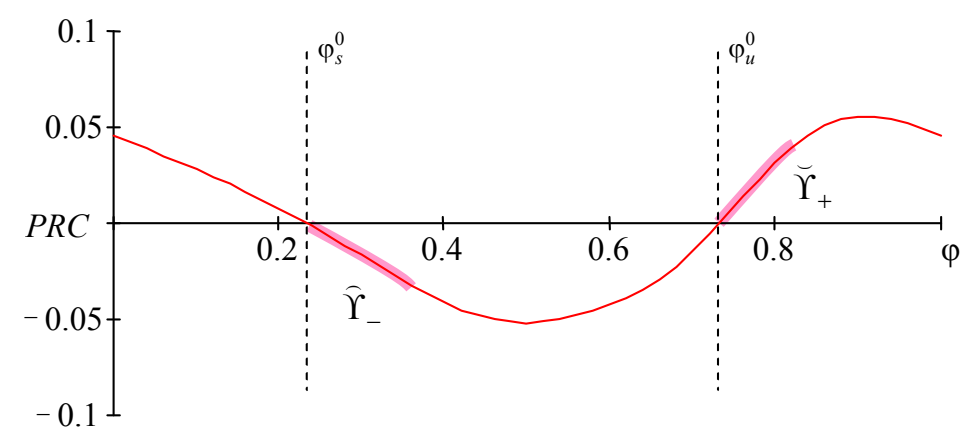

Fig. 8. PRC of the Van de Pol model.

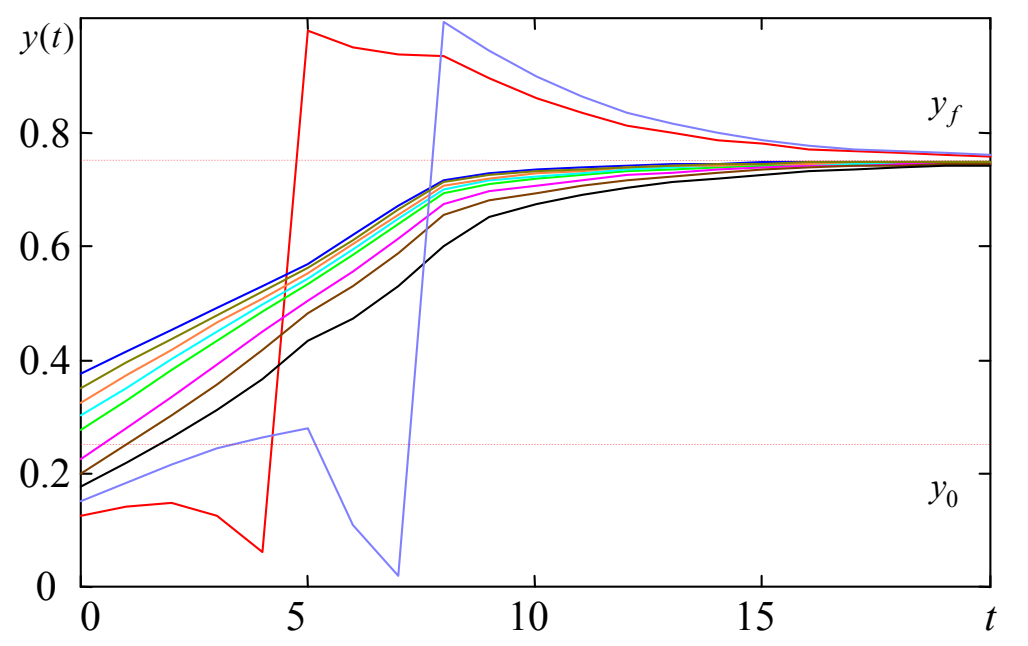

Fig. 9. The phase resetting for a network of Van der Pol models.

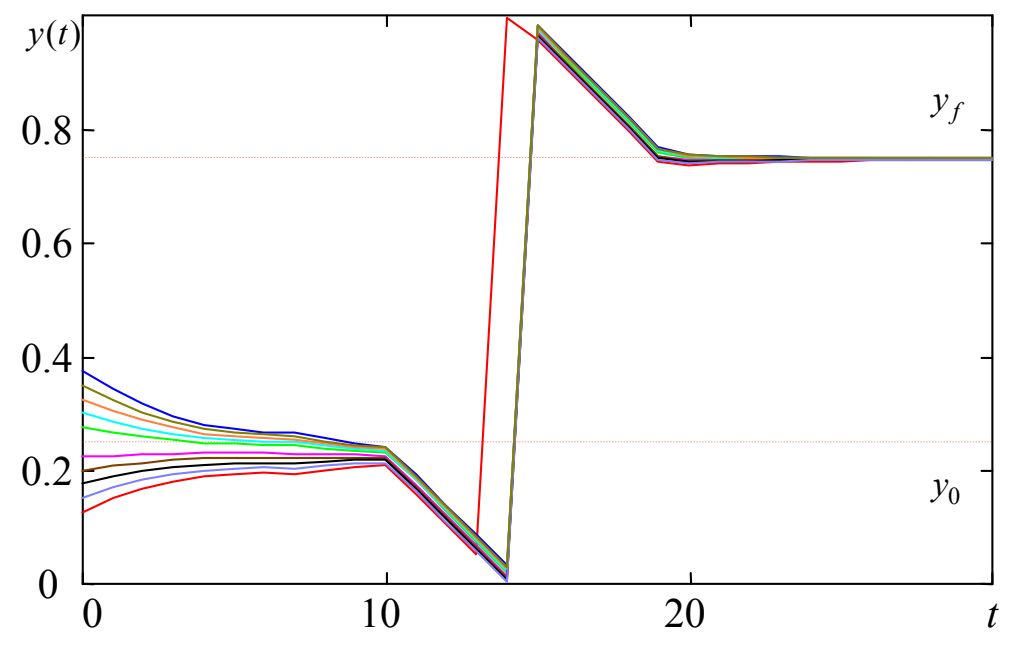

Fig. 10. The network entrainment with a periodical initial resetting.

\section{Conclusion}

A solution to the problem of phase resetting for nonlinear oscillating systems has been proposed. It is assumed that control is formed by a train of pulses of a given form and duration with adjusted instants of activation. The control is designed for a simplified PRC model of the system; this model has a discrete integrator-like form. Next, it has been proven that the control ensures practical phase resetting for the original nonlinear system (that is a contribution of the paper). Accuracy of the resetting can be managed by adjustment of the control parameters. The control robustness with respect to external disturbances and parametric fluctuations, and the influence of coupling strength on the network resetting can be analyzed in future works. A simple extension of the control algorithm is proposed, which allows a network of identical oscillators to be reset, that is confirmed by the results of simulation. Analysis of influence of 
heterogeneity of oscillators has to be performed in a future work also.

The computer simulations performed for several models of circadian oscillations confirm applicability of the proposed solution. Combination of the exponentially stabilizing control proposed in this paper and the fastest phase resetting control algorithms presented in [ESS] demonstrates the most promising results, when the controls from [ESS] provide the fastest phase resetting to a vicinity of the desired trajectory, next application of the exponentially stabilizing feedback guarantees the phase resetting error convergence for the nonlinear system.

\section{References}

AWK. Andronov A.A., Vitt A.A., Khaikin A.E. Theory of oscillators. Dover, reprint, 1987.

AFFS. Antle M.C., Foley N.C., Foley D.K., Silver R. Gates and oscillators II: zeitgebers and the network model of the brain clock. Journal of Biological Rhythms, 22, 2007, pp. 14-25.

ABS. Astashev V., Babitsky V., Kolovsky M. Dynamics and Control of Machines. New York: Springer, 2001.

BSD1. Bagheri N., Stelling J., Doyle F.J. Circadian phase entrainment via nonlinear model predictive control. Int. J. Robust and Nonlinear Control, 17, 2007, pp. 1555-1571.

BSD2. Bagheri N., Stelling J., Doyle F.J. Circadian phase resetting via single and multiple control targets. Comp. Biology, 7(4), 2008, pp. 1-10.

Be. Belykh V.N., Osipov G.V., Kucklander N., Blasius B., Kurths J. Automatic control of phase synchronization in coupled complex oscillators. Physica D, 200, 2005, pp. 81-104.

B. Blekhman I.I. Synchronization in Science and Technology. Moscow: Nauka, 1971 (in Russian) [English translation: 1988, Synchronization in Science and Technology. New York: ASME Press].

CA. Canavier C.C., Achuthan S. Pulse Coupled Oscillators and the Phase Resetting Curve. Math Biosci., 226(2), 2010, pp. 77-96.

CDST . Cheal A.J., Delean S., Sweatman H., Thompson A.A. Spatial synchrony in coral reef fish populations and the influence of climate. Ecology, 88(1), 2007, pp. 158-69.

DM. Danzl P., Moehlis J. Spike timing control of oscillatory neuron models using impulsive and quasi-impulsive charge-balanced inputs. Proc. $29^{\text {th }}$ American Control Conference (ACC2008), Seattle, USA, 2008, pp.171-176.

DS. Datta A.K., Stephens J.A. Synchronization of motor unit activity during voluntary contraction in man. J. Physiol. (Lond.), 422, 1990, pp. 397-419.

EF. Efimov D.V., Fradkov A.L. Oscillatority of Nonlinear Systems with Static Feedback. SIAM J. Optimization and Control, 48(2), 2009, pp. 618-640.

E11. Efimov D. Phase resetting control based on direct phase response curve. Journal of Mathematical Biology, 63(5), 2011, pp. 855-879.

ESS. Efimov D., Sacre P., Sepulchre R. Controlling the phase of an oscillator: a phase response curve approach. Proc. IEEE CDC 2009, 2009, pp. 7692-7697.

FP. Fradkov A.L., Pogromsky A.Y. Introduction to control of oscillations and chaos. World Scientific, 1998.

FoP. Forger D.B., Paydarfar D. Starting, stopping, and resetting biological oscillators: in search for optimum perturbations. J. Theor. Biol., 230, 2004, pp. 521-532.

Gl. Glass L., Nagai Y., Hall K., Talajic M., Nattel S. Predicting the entrainment of reentrant cardiac waves using phase resetting curves. Physical Rev. E, 65, 2002, pp. 65-74.

GS. Govaerts W., Sautois B. Computation of the Phase Response Curve: A Direct Numerical Approach. Neural Computation, 18, 2006, pp. 817-847.

GH. Guckenheimer J., Holmes P. Nonlinear Oscillations, Dynamical Systems and Bifurcations of Vector Fields. 
Springer, Berlin, 1990.

GG. Guevara M.R., Glass L. Phase locking, period doubling bifurcations and chaos in a mathematical model of a periodically driven oscillator: a theory for the entrainment of biological oscillators and the generation of cardiac dysrhythmias. J. Math Biol., 14, 1982, pp. 1-23.

GGS. Guevara M.R., Glass L., Shrier A. Phase locking, period-doubling bifurcations, and irregular dynamics in periodically stimulated cardiac cells. Science, 214, 1981, pp. 1350-1353.

HMM. Hansel D., Mato G., Meunier C. Synchrony in excitatory neural networks. Neural Comput., 7, 1995, pp. $307-337$.

I. Izhikevich E.M. Dynamical Systems in Neuroscience: The Geometry of Excitability and Bursting. The MIT press, 2007.

KS. Kawato M., Suzuki R. Biological oscillators can be stopped - Topological study of a phase response curve. Biological Cybernetics, 30(4), 1978, pp. 241-248.

KoK. Koenig W.D., Knops J.M. Large-scale spatial synchrony and cross-synchrony in acorn production by two California oaks. Ecology, 94(1), 2013, pp. 83-93.

Ko. Kovaleva A.S. Frequency and phase control of the resonance oscillations of a non-linear system under conditions of uncertainty. J. Applied Mathematics and Mechanics, 68, 2004, pp. 699-706

K. Kuramoto Y. Chemical Oscillations, Waves and Turbulence. Berlin: Springer, 1984.

Ku. Kurths J. (Ed.) A special issue on phase synchronization in chaotic systems, Int. J. Bifur. Chaos, 11, 2000.

LG98. Leloup, J.C., Goldbeter, A. A model for circadian rhythms in Drosophila incorporating the formation of a complex between the PER and TIM proteins. J. Biol. Rhythms, 13, 1998, pp. 70-87.

LG99. Leloup, J.C., Goldbeter, A. Chaos and Birhythmicity in a Model for Circadian Oscillations of the PER and TIM Proteins in Drosophila. J. Theoretical Biology, 198(3), 1999, pp. 445-459.

LGG. Leloup J.-C., Gonze D., Goldbeter A. Limit Cycle Models for Circadian Rhythms Based on Transcriptional Regulation in Drosophila and Neurospora. J. Biological Rhythms, 14, 1999, pp. 433-448.

LDR. Li J.-S., Dasanayake I., Ruths J. Control and Synchronization of Neuron Ensembles. IEEE Trans. Automat. Contr., 58(8), 2013, 1919-1930.

LSW. Lin Y., Sontag E.D. Wang Y. A Smooth Converse Lyapunov Theorem for Robust Stability. SIAM J. Control and Optimization, 34, 1996, pp. 124-160.

Mo. Moran P.A.P. The statistical analysis of the Canadian lynx cycle. II. Synchronization and meteorology. Australian Journal of Zoology, 1, 1953, pp. 291-298.

MMP. Mosekilde E., Maistrenko Yu., Postnov D. Chaotic Synchronization. Applications To Living Systems. Singapore: World Scientific, 2002.

NaN. Nakao H., Arai K., Nagai K., Tsubo Y., Kuramoto Y. Synchrony of limit-cycle oscillators induced by random external impulses. Phys. Rev. E, 72(2), 2005, p. 026220.

O. Ogata K. Discrete Time Control Systems (2 ${ }^{\text {nd }}$ Edition). Paperback, 2006.

PW. Pampus M., Winkel W. The extended Moran effect and large-scale synchronous fluctuations in the size of great tit and blue tit populations. Journal of Animal Ecology, 76, 2007, pp. 315-325.

PRK. Pikovsky A., Rosenblum M., Kurths J. Synchronization. A Universal Concept in Nonlinear Sciences. Cambridge: Cambridge University Press, 2001.

RSSM. Rand D.A., Shulgin B.V., Salazar D., Millar A.J. Design principles underlying circadian clocks. J. R. Soc. Interface, 1, 2004, pp. 119-130.

RHK. Rosenstock T.S., Hastings A., Koenig W.D., Lyles D.J. and Brown P.H. Testing Moran's theorem in an 
agroecosystem. Oikos, 120, 2011, pp. 1434-1440.

RT. Roy R., Thornburg K.S. Experimental synchronization of chaotic lasers. Phys. Rev. Lett., 72(13), 1994, pp. 20092012.

SIF. Schmied A., Ivarsson C., Fetz E.E. Short-term synchronization of motor units in human extensor digitorum communis muscle: relation to contractile properties and voluntary control. Exp. Brain Res., 97, 1993, pp. $159-172$.

SEW. Smeal R.M., Ermentrout G.B., White J.A. Phase-response curves and synchronized neural networks. Phil. Trans. R. Soc. B, 365, 2010, pp. 2407-2422.

S. Sparrow C. The Lorenz Equations: Bifurcations, Chaos and Strange Attractors. Springer-Verlag, Berlin, 1982.

T. Tass P.A. Phase Resetting in Medicine and Biology. Stochastic Modeling and Data Analysis. Berlin: Springer Verlag, 1999.

T1. Tass P.A. Desynchronization of brain rhythms with soft phase-resetting techniques. Biological Cybernetics, 87(2), 2002, pp. 102-115.

TGPD. Taylor S.R., Gunawan R., Petzold L.R., Doyle F.J. Sensitivity Measures for Oscillating Systems: Application to Mammalian Circadian Gene Network. IEEE Trans. Aut. Cont., 53(12), 2008, pp. 177-188.

TPM. Thommen Q., Pfeuty B, Morant P.-E., Corellou F., Bouget F.-Y., Lefranc M. Robustness of Circadian Clocks to Daylight Fluctuations: Hints from the Picoeucaryote Ostreococcus tauri. PLoS Comput Biol, 6(11), 2010 , e1000990.

Tr. Tran D., Nadau A., Durrieu G., Ciret P., Parisot J.C., Massabuau J.C. Field chronobiology in a molluscan bivalve: how the moon and sun cycles interact to drive oyster activity rhythms. Chronobiology International, 28, 2011, pp. 307-317.

WND. Wang Y., Núñez F., Doyle III F.J. Increasing Sync Rate of Pulse-Coupled Oscillators via Phase Response Function Design: Theory and Application to Wireless Networks. IEEE Trans. Control Systems Technology, 21(4), 2013, pp. 1455-1462.

W. Winfree A.T. The Geometry of Biological Time. Springer Verlag: Berlin, 1980.

YS. Yakubovich V.A., Starzhinskii V.M. Linear differential equations with periodic coefficients. Wiley, 1975.

Z. Zhao G. Phase organization of circadian oscillators in extended gate and oscillator models. J. Theoretical Biology, 264(2), 2010, pp. 367-376. 